\title{
Mapping 123 million neonatal, infant and child deaths between 2000 and 2017
}

\author{
A list of authors and their affiliations appears in the online version of the paper.
}

Since 2000, many countries have achieved considerable success in improving child survival, but localized progress remains unclear. To inform efforts towards United Nations Sustainable Development Goal 3.2-to end preventable child deaths by 2030 -we need consistently estimated data at the subnational level regarding child mortality rates and trends. Here we quantified, for the period 2000-2017, the subnational variation in mortality rates and number of deaths of neonates, infants and children under 5 years of age within 99 low- and middle-income countries using a geostatistical survival model. We estimated that $32 \%$ of children under 5 in these countries lived in districts that had attained rates of 25 or fewer child deaths per 1,000 live births by 2017 , and that $58 \%$ of child deaths between 2000 and 2017 in these countries could have been averted in the absence of geographical inequality. This study enables the identification of high-mortality clusters, patterns of progress and geographical inequalities to inform appropriate investments and implementations that will help to improve the health of all populations.

Gains in child survival have long served as an important proxy measure for improvements in overall population health and development ${ }^{1,2}$. Global progress in reducing child deaths has been heralded as one of the greatest success stories of global health ${ }^{3}$. The annual global number of deaths of children under 5 years of age (under 5$)^{4}$ has declined from 19.6 million in 1950 to 5.4 million in 2017. Nevertheless, these advances in child survival have been far from universally achieved, particularly in low- and middle-income countries (LMICs) ${ }^{4}$. Previous subnational child mortality assessments at the first (that is, states or provinces) or second (that is, districts or counties) administrative level indicate that extensive geographical inequalities persist ${ }^{5-7}$.

Progress in child survival also diverges across age groups ${ }^{4}$. Global reductions in mortality rates of children under 5-that is, the under-5 mortality rate (U5MR) - among post-neonatal age groups are greater than those for mortality of neonates $(0-28 \text { days })^{4,8}$. It is relatively unclear how these age patterns are shifting at a more local scale, posing challenges to ensuring child survival. To pursue the ambitious Sustainable Development Goal (SDG) of the United Nations" to "end preventable deaths of newborns and children under 5 " by 2030 , it is vital for decision-makers at all levels to better understand where, and at what ages, child survival remains most tenuous.

\section{Precision public health and child mortality}

Country-level estimates facilitate international comparisons but mask important geographical heterogeneity. Previous assessments of mortality of children under 5 have noted significant within-country heterogeneity, particularly in sub-Saharan Africa ${ }^{5,7,10-14}$, as well as in Brazil ${ }^{15}$, Iran $^{16}$ and China ${ }^{17}$. Understanding public health risks at more granular subpopulation levels is central to the emerging concept of precision public health ${ }^{18}$, which uses "the best available data to target more effectively and efficiently interventions... to those most in need"18. Efforts to produce high-resolution estimates of mortality of children under 5 , determinants at scales that cover the multiple countries are emerging, including for vaccine coverage ${ }^{19,20}$, malaria ${ }^{21}$, diarrhoea ${ }^{22}$ and child growth failure ${ }^{23,24}$. In a previous study, we produced comprehensive estimates of African child mortality rates at a $5 \times 5-\mathrm{km}$ scale for 5 -year intervals ${ }^{5}$. For areas outside of Africa, in which $72 \%$ of the world's children live and $46 \%$ of global child deaths occurred in $2017^{4}$, subnational heterogeneity remains mostly undescribed ${ }^{25}$.

Here we produce estimates of death counts and mortality rates of children under 5 , infants (under 1 years of age) and neonates (0-28 days) in 99 countries at policy-relevant subnational scales (first and second administrative levels) for each year from 2000 to 2017 . We fit a geostatistical discrete hazards model to a large dataset that is composed of 467 geo-referenced household surveys and censuses, representing approximately 15.9 million births and 1.1 million deaths of children from 2000 to 2017. Our model includes socioeconomic, environmental and health-related spatial covariates with known associations to child mortality and uses a Gaussian process random effect to exploit the correlation between data points near each other across dimensions of space, time and age group, which helps to mitigate the limitations associated with data sparsity in our estimations. For this study, we report U5MR as the expected number of deaths per 1,000 live births, reflecting the probability of dying before the age of 5 for a given location and year.

\section{Unequal rates of child mortality}

The risk of a newborn dying before their fifth birthday varies tremendously based on where in the world, and within their country, they are born. Across the 99 countries in this study, we estimate that U5MR varied as much as 24-fold at the national level in 2017, with the highest rate in the Central African Republic of 123.9 deaths (95\% uncertainty interval, 104.9-148.2) per 1,000 live births, and the lowest rate in Cuba of 5.1 deaths $(4.4-6.0)^{4}$. We observed large subnational variation within countries in which overall U5MR was either high or comparatively low. For example, in Vietnam, rates across second administrative units (henceforth referred to as 'units') varied 5.7-fold, from 6.9 (4.6-9.8) in the Tenth District in Hồ Chí Minh City to 39.7 (28.1-55.6) in Mường Tè District in the Northwest region (Figs. 1b, 2).

Decreases in U5MR between 2000 and 2017 were evident to some extent throughout all units (Figs. 1a, b, 2). No unit showed a significant increase in U5MR in this period, and in most units U5MR decreased greatly, even in units in which the mortality risk was the highest. Out of 17,554 units, $60.3 \%$ (10,585 units) showed a significant (defined as $95 \%$ uncertainty intervals that did not overlap) decrease in U5MR between 2000 and 2017. Across units in 2000, U5MR ranged from 7.5 (5.0-10.6) in Santa Clara district, Villa Clara province, Cuba, to 308.4 (274.9-348.4) in the Sabon Birni Local Government Area of Sokoto State, Nigeria. By 2017, the unit with the highest estimated U5MR across all 99 countries was Garki Local Government Area, Jigawa state, Nigeria, at 195.1 (158.6-230.9). Overall, the total percentage of units with a U5MR higher than 80 deaths per 1,000 live births decreased from $28.9 \%(5,070)$ of units in 2000 to $7.0 \%(1,236)$ in 2017 . Furthermore, 

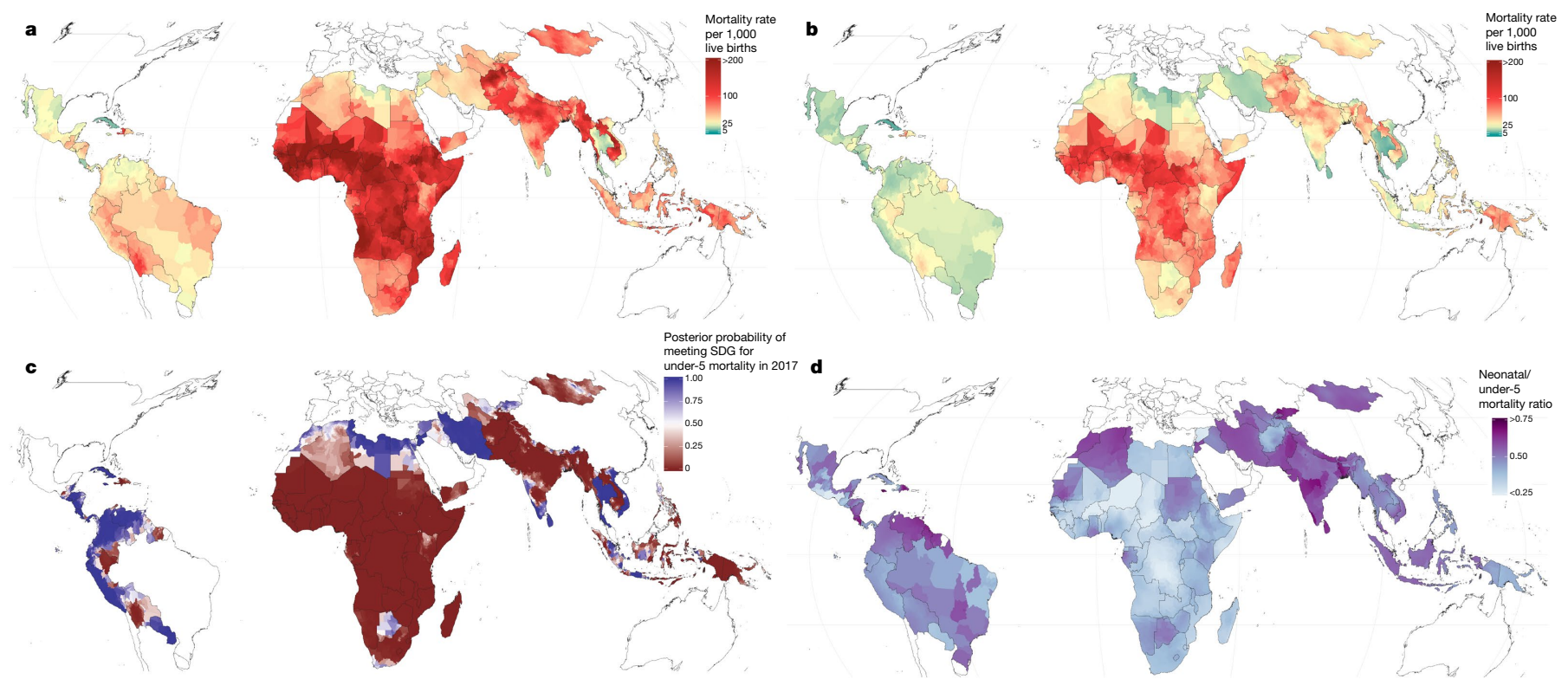

Fig. 1 | U5MR estimates in 99 LMICs. a, U5MR at the second administrative level in 2000. b, U5MR at the second administrative level in 2017. c, Modelled posterior exceedance probability that a given second administrative unit had achieved the SDG 3.2 target of 25 deaths per

$32 \%$ of units, representing $11.9 \%$ of the under-5 population in the 99 countries, had already met SDG 3.2 for U5MR with a $90 \%$ certainty threshold (Fig. 1c). For neonatal mortality, $34 \%$ of units met the target of $\leq 12$ deaths per 1,000 live births (Extended Data Fig. 1). Within countries, successes were mixed in some cases. For example, Colombia, Guatemala, Libya, Panama, Peru and Vietnam had all achieved SDG 3.2 for U5MR at the national level by 2017, but each country had units that did not achieve the goal with $90 \%$ certainty (Fig. 1c).

Successful reductions in child mortality were also observed throughout entire countries. For example, in 43 LMICs across several world regions, the worst-performing unit in 2017 had a U5MR that was lower than the best-performing unit in 2000 (Fig. 2). Nearly half of these countries were in sub-Saharan Africa. Rwanda showed notable progress during the study period, reducing mortality from 144.0 (130.0-161.6) in its best-achieving district in 2000 (Rubavu) to 57.2 (47.4-72.1) in its worst-achieving district in 2017 (Kayonza). These broad reductions in U5MR have also led to a convergence of absolute subnational geographical inequalities, although relative subnational inequalities appear to be mostly unchanged between 2000 and 2017 (Fig. 2 and Supplementary Fig. 6.12). Despite this success, the highest U5MRs in 2017 were still largely concentrated in areas in which rates were highest in 2000 (Fig. 1a, b). We observed estimated U5MR $\geq 80$ across large geographical areas in Western and Central sub-Saharan Africa, and within Afghanistan, Cambodia, Haiti, Laos and Myanmar (Fig. 1b).

Deaths of neonates (0-28 days of age) and post-neonates (28-364 days of age) have come to encompass a larger fraction of overall mortality of children under 5 in recent years. By 2017 (Fig. 1d), neonatal mortality increased as a proportion of total deaths of children under 5 in $91 \%$ (90) of countries and for $83 \%(14,656)$ of units compared to 2000. In almost all places where U5MR decreased, the share of the mortality burden increased in the groups of children with younger ages. Similarly, the mortality of infants ( $<1$ year) has increased relative to the mortality for children who are 1-4 years of age in many areas. For example, in the Diourbel Region, Senegal, infant mortality constituted $54.4 \%$ (52.4-56.6) of total mortality of children under 5 in 2000; by 2017 , the relative contribution of infant mortality was $73.2 \%$ (70.375.8). This shift towards mortality predominantly affecting neonates and infants was not as evident in all locations; mortality for children aged 1-4 years was responsible for more than $30 \%$ of overall under-5
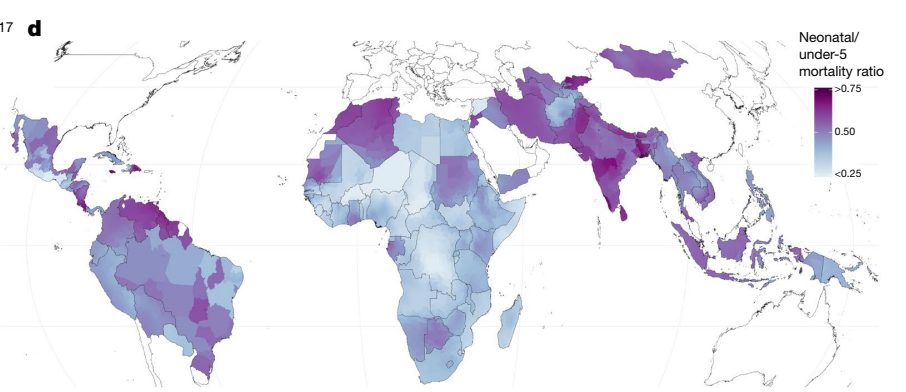

1,000 live births for children under 5 in 2017. d, Proportion of mortality of children under 5 occurring in the neonatal (0-28 days) group at the second administrative level in 2017.

deaths in $13 \%(2,226)$ of units, mostly within high-mortality areas in sub-Saharan Africa.

\section{Distribution of under-5 deaths may not follow rates}

The goal of mortality-reduction efforts is ultimately to prevent premature deaths, and not just to reduce mortality rates. Across the countries studied here, there were 3.5 million (41\%) fewer deaths of children under 5 in 2017 than in 2000 (5.0 million compared to 8.5 million). At the national level, the largest number of child deaths in 2017 occurred in India (1.04 (0.98-1.10) million), Nigeria (0.79 (0.65-0.96) million), Pakistan (0.34 (0.27-0.41) million) and the Democratic Republic of the Congo (0.25 (0.21-0.31) million) (Fig. 3a). Within these countries, the geographical concentration of the deaths of the children varied. In Pakistan, over 50\% of child deaths in 2017 occurred in Punjab province, which had a U5MR of 63.3 (54.1-76.0) deaths per 1,000 live births (Fig. 3b). By contrast, $50 \%$ of child deaths in the Democratic Republic of the Congo in 2017 occurred across 9 out of 26 provinces. Such findings are in a large part artefacts of how borders are drawn around various at-risk populations (the provinces above account for $53 \%$ and $63 \%$, respectively, of the under-5 population that is at risk in these two countries), but can have a real impact at the level at which planning occurs. Some concentrated areas with apparent high absolute numbers of deaths highlighted by local-level estimates become less noticeable when reporting at aggregated administrative levels; for example, areas across Guatemala, Honduras and El Salvador are visually striking hotspots in Fig. 3d, but less so in Fig. 3b, c.

Our estimates indicate that targeting areas with a 'high' U5MR of 80 will have a lower overall effect than in previous years owing to the reductions in mortality rates. In $2000,23.7 \%$ of child deathsrepresenting 2.0 (1.7-2.4) million deaths-occurred in regions in which U5MR was less than 80 that year (Fig. 4). By comparison, in 2017, $69.5 \%$ of child deaths occurred in areas in which U5MR was below 80 . A growing proportion of deaths of children under 5 are occurring in 'low'-mortality areas; 7.3\% (5.1-10.2) of all deaths of children under 5 in 2017 occurred in locations in which the U5MR was below the SDG 3.2 target rate of 25 , compared to $1.2 \%(0.9-1.6)$ in 2000 . For instance, Lima, Peru, has a U5MR in the 8th percentile of units in this study, yet it ranks in the 96th percentile of highest number of deaths of children under 5. 
a
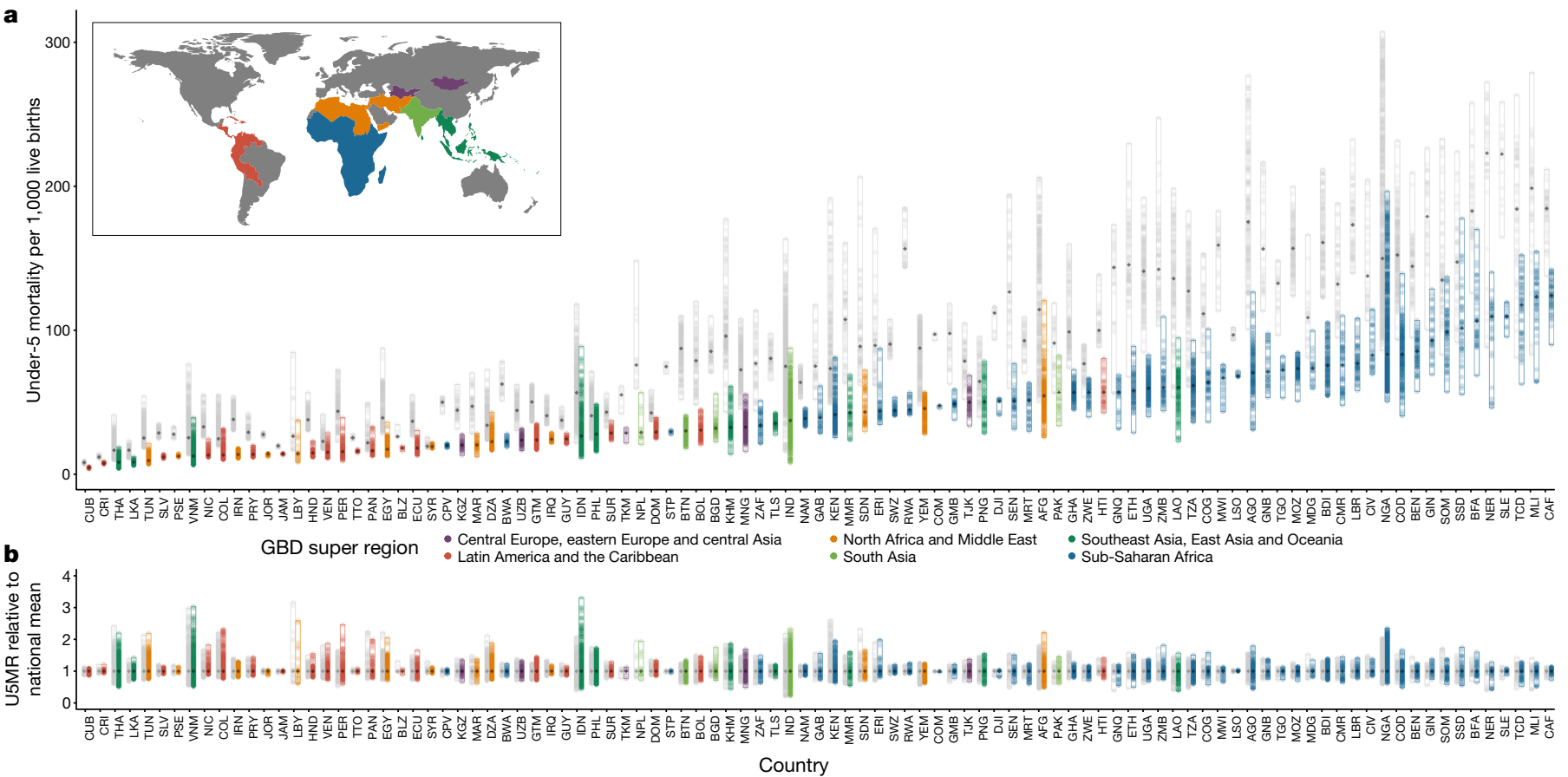

Fig. 2 Geographical inequality in U5MR across 99 countries for 2000 and 2017. a, Absolute inequalities. Range of U5MR estimates in second administrative-level units across 99 LMICs. b, Relative inequalities. Range of ratios of U5MR estimates in second administrative-level units relative to country means. Each dot represents a second administrative-level unit. The lower bound of each bar represents the second administrative-level unit with the lowest U5MR in each country. The upper end of each bar represents the second administrative-level unit with the highest U5MR

Despite population growth, child deaths have declined due to the outpaced decline in U5MR. For example, there were a total of 8.5 (7.2-10.0) million deaths of children under 5 in the countries in this study in 2000; had the 2017 under-5 population been exposed to the same U5MRs that were observed in 2000, there would have been 10.6 (9.0-12.5) million deaths in 2017. Instead, we observed 5.0 (3.8-6.6) million deaths in 2017 (Extended Data Fig. 5).

Finally, we combine estimates of subnational variation in mortality rates and populations to gain a better understanding of the impact of geographical inequality. Overall, 2.7 (2.5-2.9) million deaths, or $54 \%$ of the total number of deaths of children under 5 , would have been averted in 2017 had all units had a U5MR that matched the bestperforming unit in each respective country (Extended Data Fig. 2). Over the 2000-2017 period, this number is 71.8 (68.5-74.9) million deaths, or $58 \%$ (55-61) of the total number of deaths of children under 5 . Total deaths attributable to inequality in this scenario ranged from 13 (6-24) deaths in Belize to 0.84 (0.72-0.99) million deaths in India. Furthermore, had all units met the SDG 3.2 target of 25 deaths per 1,000 , an estimated $2.6(2.3-2.8)$ million deaths of children under 5 would have been averted in 2017.

\section{Discussion}

This study offers a comprehensive, geospatially resolved resource for national and subnational estimates of child deaths and mortality rates for 99 LMICs, where $93 \%$ of the world's child deaths ${ }^{4}$ occurred in 2017. Gains in child survival varied substantially within the vast majority of countries from 2000 to 2017. Countries such as Vietnam, for example, showed more than fivefold variation in mortality rates across second administrative-level units. The inconsistency of successes, even at subnational levels, indicates how differences in health policy, financial resources, access to and use of health services, infrastructure, and economic development ultimately contribute to millions of lives cut short $^{25-27}$. By providing detailed maps that show precisely where these in each country. Thus, each bar represents the extent of geographical inequality in U5MRs estimated for each country. Bars indicating the range in 2017 are coloured according to their Global Burden of Disease super-region. Grey bars indicate the range in U5MR in 2000. The diamond in each bar represents the median U5MR estimated across second administrative-level units in each country and year. A coloured bar that is shorter than its grey counterpart indicates that geographical inequality has narrowed.

deaths are estimated to have occurred, we provide an important evidence base for looking both to the past, for examples of success, and towards the future, in order to identify where precision public-health initiatives could save the most lives.

The epidemiological toll of child mortality should be considered both in terms of total deaths and as rates of mortality. Focusing only on mortality rates can effectively mask areas in which rates are comparatively low but child deaths are high owing to large population sizes. The number of deaths that occur in high-risk areas has declined, and most under-5 deaths in recent years have occurred in lower-risk areas. This 'prevention paradox ${ }^{28}$ could indicate that whole-population interventions could have a larger overall impact than targeting high-risk areas ${ }^{29}$. At the same time, strategies that target resources to those locations that have the highest number of child deaths risk leaving behind some of the world's most marginalized communities: remote, more-sparsely populated places in which, relative to the number of children born each year, a large number of children die before their fifth birthday. Instead, by considering subnational measures of both counts and rates of deaths of children under 5 , decision-makers can better tailor child health programs to align with local contexts, norms and needs. Rural communities with high rates but low counts may benefit from 'lastmile' initiatives to provide effective health services to populations who lack adequate access to care. By contrast, locations with low rates but high counts may require programs that focus on alleviating the cost of care, unsafe environmental exposures or health risks that are uniquely associated with urban slums ${ }^{30}$. The SDGs have pointed the global development agenda towards progress in child survival. Our analysis indicates that reaching the SDG 3.2 targets of 25 child deaths per 1,000 live births and 12 neonatal deaths per 1,000 live births will require only modest improvements or have already been achieved by some units; however, these targets are ambitious for other units in which child mortality remains high. It is worth noting that many countries contain areas that fit both of these profiles. For example, 11 countries 

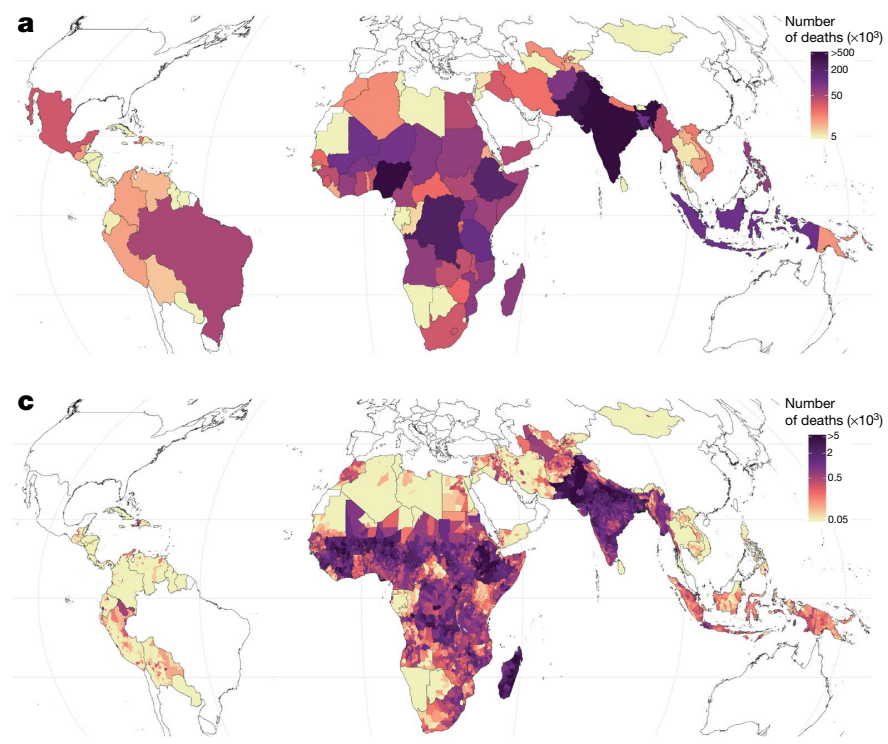

Fig. 3 Estimated number of children under 5 who died within 99 countries in 2017. a, Number of deaths of children under 5 in each country. b, Number of deaths in each first administrative-level unit.

had at least 1 unit that had already met SDG 3.2 with high certainty, and at least 1 unit that had not. Subnational estimates can empower countries to benchmark gains in child survival against their own subnational exemplars as well as advances that have been achieved by their peers. Through our counterfactual analysis we showed that even if all units had met the SDG 3.2 goal in 2017, there would still have been 2.4 million deaths of children under 5 , indicating that 'ending preventable child deaths' is more complex than simply meeting a target threshold. Future research efforts must address the causes of child mortality in local areas and more precisely identify causes of child deaths that are amenable to intervention. To that end, new and innovative data-collection efforts, such as the ongoing Child Health and Mortality Prevention Surveillance network, offer promising prospects by applying high-validity, pathology-based methods alongside verbal autopsies to determine the cause of death ${ }^{31}$.

This study offers a unique platform to support the identification of local success stories that could be replicated elsewhere. In Rwanda, for example, the highest U5MR at the district level in 2017 was $60.2 \%$ (52.0-67.8\%) lower than the lowest U5MR at the district level in 2000. Such gains have been partially credited to focused investments in the country's poorest populations, expanding the Mutuelles de santé insurance program, and developing a strong workforce of community health workers who provide evidence-based treatment and health promotion $^{32,33}$. Nepal and Cambodia are among the exemplars for considerably decreasing subnational inequalities in child survival since 2000. In an era when narrowing disparities within countries is as important as reducing national-level gaps, these results provide the evidence base to inform best practices and stimulate national conversations about related social determinants.

Neonatal mortality rates have also declined but failed to keep pace with reductions in mortality rates of older children, leading to a higher proportion of deaths of children under 5 occurring within the first four weeks of life: from $37.4 \%$ (37.1-37.7) in 2000 to $43.7 \%$ (43.1-44.3\%) in 2017. This trend is probably related to the increase in scale of routine programs and improved infrastructure (for example, vaccination ${ }^{34}$, and water and sanitation ${ }^{35}$ ) and the introduction of effective interventions to target communicable diseases (for example, malaria control ${ }^{36}$ and prevention of mother-to-child transmission of $\mathrm{HIV}^{37}$ ). These interventions have tended to target amenable causes of mortality that are more common in older children under 5 rather than dominant causes of neonatal mortality, such as prematurity and congenital anomalies ${ }^{38}$.
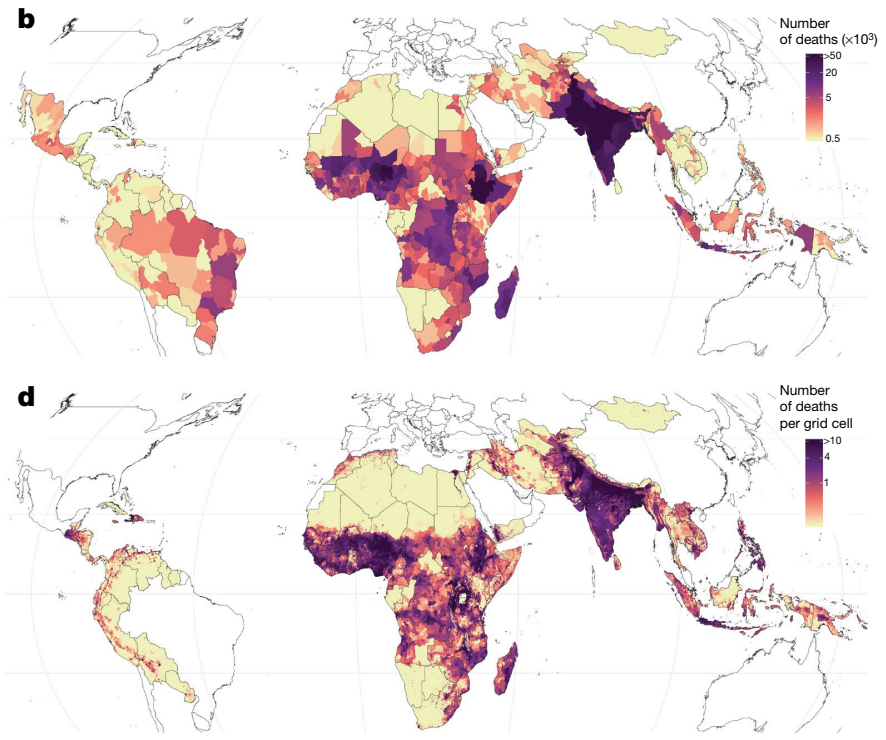

c, Number of deaths in each second administrative-level unit. d, Number of deaths of children under 5 in each $5 \times 5-\mathrm{km}$ grid cell. Note that scales vary for each aggregation unit.

Notably, irrespective of income level or location, some causes of neonatal death (for example, chromosomal anomalies and severe preterm birth complications) remain difficult to prevent completely with current medical technologies. Ultimately, large gains in neonatal mortality will require serious investment in health system strengthening ${ }^{39}$. Affordable approaches to preventing the majority of neonatal deaths in LMICs exist and there are success stories with lessons learned to apply ${ }^{40-44}$, but decisions about which approaches to take must be based on the local epidemiological and health system context. In the absence of spatially detailed cause of death data, subnational neonatal mortality estimates can indicate dominant causes and thus serve as a useful proxy to guide prioritization of interventions $s^{45}$.

The accuracy and precision of our estimates were primarily determined by the timeliness, quantity and quality of available data. In Sri Lanka, for example, there were no available surveys, and the wide uncertainty intervals surrounding estimates reflect the dearth of available evidence in that country (Extended Data Figs. 3, 4). In certain areas, this decreased the confidence that we had in claiming that a specific subnational area met the SDG 3.2 target (Fig. 1c). This issue is most concerning in cases in which estimated mortality rates are high, thus helping to identify locations in which it would be most useful to focus future data-collection efforts. High mortality rates with large uncertainty intervals were estimated across much of Eastern and Central sub-Saharan Africa, and in Cambodia, Laos, Myanmar and Papua New Guinea (Extended Data Figs. 3, 4). Furthermore, ongoing conflict in countries such as Syria, Yemen and Iraq pose substantial challenges to collecting more contemporaneous data, and our estimates may not fully capture the effects of prolonged civil unrest or war ${ }^{46,47}$. Further methodological and data limitations are discussed in the Methods.

The accurate estimation of mortality is also a matter of equity; highly refined health surveillance is common in high-income countries, whereas in LMICs, in which rates of child mortality are the highest, surveillance that helps to guide investments in health towards the areas with the greatest need is less routine ${ }^{48}$. Ideally, all countries would have high-quality, continuous, and complete civil and vital registration systems that capture all of the births, deaths and causes of death at the appropriate geographical resolution ${ }^{49}$. In the meantime, analyses such as this serve to bridge the information gap that exists between low-mortality countries with strong information systems and countries that face a dual challenge of weaker information systems and higher disease burden. 

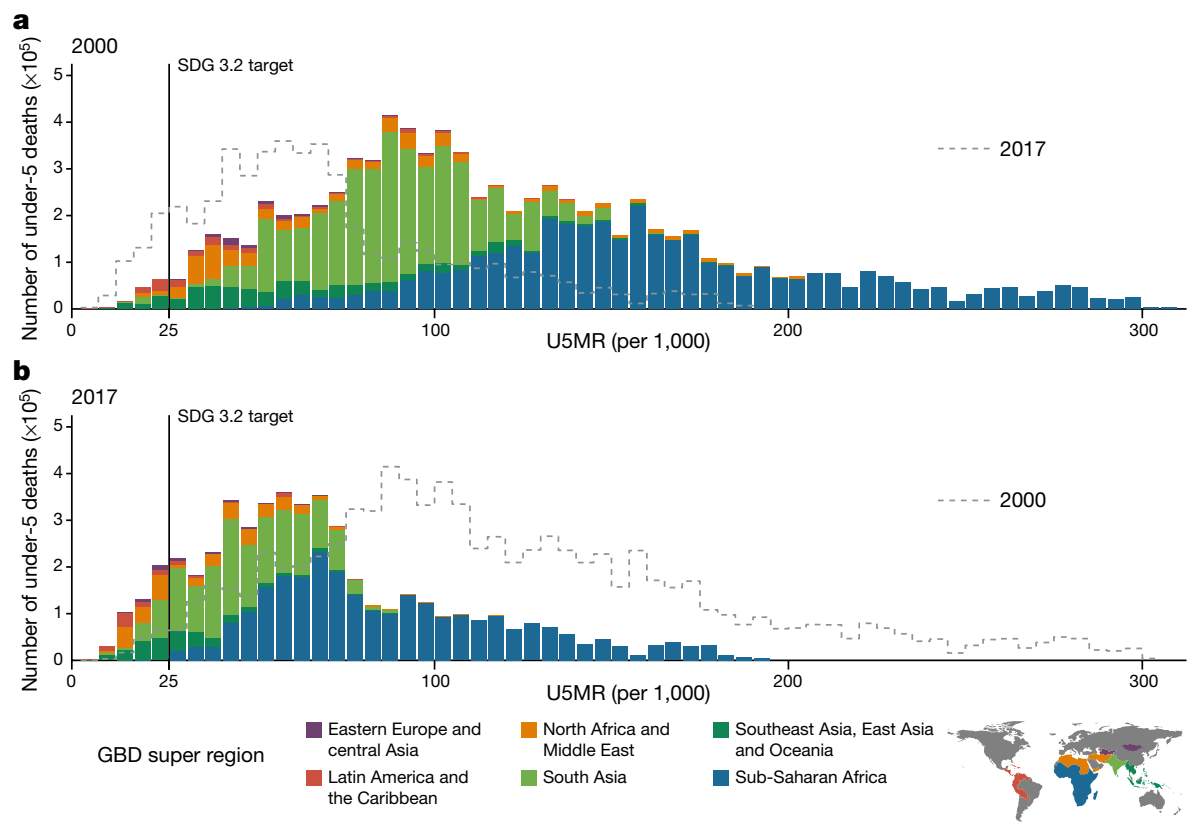

Fig. 4 | Number of deaths of children under 5, distributed across level of U5MR, in 2000 and in 2017, across 99 countries. Bar heights represent the total number of deaths of children under 5 within all second administrative-level units with corresponding U5MR. Bins are a width of 5 deaths per 1,000 live births. The colour of each bar represents the global region as defined by the subset legend map. As such, the sum of heights

By harnessing the unprecedented availability of geo-referenced data and developing robust statistical methods, we provide a high-resolution atlas of child death counts and rates since 2000, covering countries that account for $93 \%$ of child deaths. We bring attention to subnational geographical inequalities in the distribution, rates and absolute counts of child deaths by age. These high-resolution estimates can help decision-makers to structure policy and program implementation and facilitate pathways to end preventable child deaths ${ }^{50}$ by 2030 .

\section{Online content}

Any methods, additional references, Nature Research reporting summaries, source data, extended data, supplementary information, acknowledgements, peer review information; details of author contributions and competing interests; and statements of data and code availability are available at https://doi.org/10.1038/s41586-019-1545-0.

Received: 28 March 2019; Accepted: 6 August 2019;

Published online 16 October 2019.

1. Reidpath, D. D. \& Allotey, P. Infant mortality rate as an indicator of population health. J. Epidemiol. Community Health 57, 344-346 (2003).

2. United Nations General Assembly. United Nations Millennium Declaration: Resolution adopted by the General Assembly. A/RES/55/2 (UN General Assembly, 2000).

3. Centers for Disease Control and Prevention. Ten Great Public Health Achievements—Worldwide, 2001-2010. https://www.cdc.gov/mmwr/preview/ $\mathrm{mmwrhtml} / \mathrm{mm} 6024 \mathrm{a} 4 . \mathrm{htm}$ (2011).

4. GBD 2017 Mortality Collaborators. Global, regional, and national age-sexspecific mortality and life expectancy, 1950-2017: a systematic analysis for the Global Burden of Disease Study 2017. Lancet 392, 1684-1735 (2018).

5. Golding, N. et al. Mapping under-5 and neonatal mortality in Africa, 2000-15: a baseline analysis for the Sustainable Development Goals. Lancet 390, 2171-2182 (2017).

6. Pezzulo, C. et al. Geospatial Modeling of Child Mortality across 27 Countries in sub-Saharan Africa. DHS Spatial Analysis Reports No. 13 (USAID, 2016).

7. Li, Z. et al. Changes in the spatial distribution of the under-five mortality rate: small-area analysis of 122 DHS surveys in 262 subregions of 35 countries in Africa. PLOS ONE 14, e0210645 (2019).

8. Lawn, J. E., Cousens, S. \& Zupan, J. 4 million neonatal deaths: When? Where? Why? Lancet 365, 891-900 (2005)

9. World Health Organization. SDG 3: Ensure healthy lives and promote wellbeing for all at all ages. https://www.who.int/sdg/targets/en/ (2019)

10. Dwyer-Lindgren, L. et al. Estimation of district-level under-5 mortality in Zambia using birth history data, 1980-2010. Spat. SpatioTemporal Epidemiol. 11, 89-107 (2014). of all bars represents the total number of deaths across the 99 countries. a, Deaths of children under 5 in 2000. b, Deaths of children under 5 in 2017. The dotted line in the 2000 plot is the shape of the distribution in 2017, and the dotted line in the 2017 plot represents the distribution in 2000.

11. Dwyer-Lindgren, L. et al. Small area estimation of under- 5 mortality in Bangladesh, Cameroon, Chad, Mozambique, Uganda, and Zambia using spatially misaligned data. Popul. Health Metr. 16, 13 (2018).

12. Wakefield, J. et al. Estimating under-five mortality in space and time in a developing world context. Stat. Methods Med. Res. https://doi. org/10.1177/0962280218767988 (2018).

13. Burke, M., Heft-Neal, S. \& Bendavid, E. Sources of variation in under- 5 mortality across sub-Saharan Africa: a spatial analysis. Lancet Glob. Health 4, e936-e945 (2016).

14. Macharia, P. M. et al. Sub national variation and inequalities in under-five mortality in Kenya since 1965. BMC Public Health 19, 146 (2019).

15. Sousa, A., Hill, K. \& Dal Poz, M. R. Sub-national assessment of inequality trends in neonatal and child mortality in Brazil. Int. J. Equity Health 9, 21 (2010).

16. Mohammadi, Y. et al. Measuring Iran's success in achieving Millennium Development Goal 4: a systematic analysis of under-5 mortality at national and subnational levels from 1990 to 2015. Lancet Glob. Health 5, e537-e544 (2017).

17. Wang, Y. et al. Under-5 mortality in 2851 Chinese counties, 1996-2012: a subnational assessment of achieving MDG 4 goals in China. Lancet 387, 273-283 (2016)

18. Horton, R. Offline: in defence of precision public health. Lancet 392, 1504 (2018)

19. Takahashi, S., Metcalf, C. J. E., Ferrari, M. J., Tatem, A. J. \& Lessler, J. The geography of measles vaccination in the African Great Lakes region. Nat. Commun. 8, 15585 (2017)

20. Utazi, C. E. et al. High resolution age-structured mapping of childhood vaccination coverage in low and middle income countries. Vaccine $\mathbf{3 6}$, 1583-1591 (2018).

21. Gething, P. W. et al. Mapping Plasmodium falciparum mortality in Africa between 1990 and 2015. N. Engl. J. Med. 375, 2435-2445 (2016).

22. Reiner, R. C. Jr et al. Variation in childhood diarrheal morbidity and mortality in Africa, 2000-2015. N. Engl. J. Med. 379, 1128-1138 (2018).

23. Osgood-Zimmerman, A. et al. Mapping child growth failure in Africa between 2000 and 2015. Nature 555, 41-47 (2018).

24. Amoah, B., Giorgi, E., Heyes, D. J., van Burren, S. \& Diggle, P. J. Geostatistical modelling of the association between malaria and child growth in Africa. Int. J. Health Geogr. 17, 7 (2018).

25. Bishai, D. M. et al. Factors contributing to maternal and child mortality reductions in 146 low- and middle-income countries between 1990 and 2010 PLOS ONE 11, e0144908 (2016).

26. Marmot, M. Social determinants of health inequalities. Lancet 365, 1099-1104 (2005).

27. Victora, C. G. et al. Countdown to 2015: a decade of tracking progress for maternal, newborn, and child survival. Lancet 387, 2049-2059 (2016).

28. Rose, G. Strategy of prevention: lessons from cardiovascular disease. Br. Med. J. (Clin. Res. Ed.) 282, 1847-1851 (1981).

29. Mackenbach, J. P., Lingsma, H. F., van Ravesteyn, N. T. \& Kamphuis, C. B. M. The population and high-risk approaches to prevention: quantitative estimates of their contribution to population health in the Netherlands, 1970-2010. Eur. J. Public Health 23, 909-915 (2013). 
30. Agarwal, S. \& Taneja, S. All slums are not equal: child health conditions among the urban poor. Indian Pediatr. 42, 233-244 (2005).

31. Farag, T. H. et al. Precisely tracking childhood death. Am. J. Trop. Med. Hyg. 97, 3-5 (2017).

32. Farmer, P. E. et al. Reduced premature mortality in Rwanda: lessons from success. Br. Med. J. 346, f65 (2013).

33. Gurusamy, P. S. R. \& Janagaraj, P. D. A success story: the burden of maternal, neonatal and childhood mortality in Rwanda - critical appraisal of interventions and recommendations for the future. Afr. J. Reprod. Health 22, 9-16 (2018).

34. McGovern, M. E. \& Canning, D. Vaccination and all-cause child mortality from 1985 to 2011: global evidence from the Demographic and Health Surveys. Am. J. Epidemiol. 182, 791-798 (2015).

35. Cheng, J. J., Schuster-Wallace, C. J., Watt, S., Newbold, B. K. \& Mente, A. An ecological quantification of the relationships between water, sanitation and infant, child, and maternal mortality. Environ. Health 11, 4 (2012).

36. Steketee, R. W. \& Campbell, C. C. Impact of national malaria control scale-up programmes in Africa: magnitude and attribution of effects. Malar. J. 9, 299 (2010).

37. Kiragu, K., Collins, L., Von Zinkernagel, D. \& Mushavi, A. Integrating PMTCT into maternal, newborn, and child health and related services: experiences from the global plan priority countries. J. Acquir. Immune Defic. Syndr. 75, S36-S42 (2017).

38. GBD 2017 Causes of Death Collaborators. Global, regional, and national age-sex-specific mortality for 282 causes of death in 195 countries and territories, 1980-2017: a systematic analysis for the Global Burden of Disease Study 2017. Lancet 392, 1736-1788 (2018)

39. Pasha, O. et al. A combined community- and facility-based approach to improve pregnancy outcomes in low-resource settings: a Global Network cluster randomized trial. BMC Med. 11, 215 (2013).

40. Horton, S. et al. Ranking 93 health interventions for low- and middle-income countries by cost-effectiveness. PLoS ONE 12, e0182951 (2017).

41. Simmons, L. E., Rubens, C. E., Darmstadt, G. L. \& Gravett, M. G. Preventing preterm birth and neonatal mortality: exploring the epidemiology, causes, and interventions. Semin. Perinatol. 34, 408-415 (2010).

42. Darmstadt, G. L. et al. Evidence-based, cost-effective interventions: how many newborn babies can we save? Lancet 365, 977-988 (2005)

43. Saugstad, O. D. Reducing global neonatal mortality is possible. Neonatology 99 250-257 (2011).
44. Ntigurirwa, P. et al. A health partnership to reduce neonatal mortality in four hospitals in Rwanda. Glob. Health 13, 28 (2017).

45. Knippenberg, R. et al. Systematic scaling up of neonatal care in countries. Lancet 365, 1087-1098 (2005).

46. GBD 2015 Eastern Mediterranean Region Neonatal, Infant, and under-5 Mortality Collaborators. Neonatal, infant, and under-5 mortality and morbidity burden in the Eastern Mediterranean region: findings from the Global Burden of Disease 2015 study. Int. J. Public Health 63, 63-77 (2018).

47. Wagner, Z. et al. Armed conflict and child mortality in Africa: a geospatial analysis. Lancet 392, 857-865 (2018).

48. Mikkelsen, L. et al. A global assessment of civil registration and vital statistics systems: monitoring data quality and progress. Lancet 386, 1395-1406 (2015).

49. AbouZahr, $C$ et al. Civil registration and vital statistics: progress in the data revolution for counting and accountability. Lancet 386, 1373-1385 (2015).

50. Annan, K. Data can help to end malnutrition across Africa. Nature 555, 7 (2018).

Publisher's note Springer Nature remains neutral with regard to jurisdictional claims in published maps and institutional affiliations.

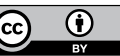

Open Access This article is licensed under a Creative Commons Attribution 4.0 International License, which permits use, sharing, adaptation, distribution and reproduction in any medium or format, as long as you give appropriate credit to the original author(s) and the source, provide a link to the Creative Commons license, and indicate if changes were made. The images or other third party material in this article are included in the article's Creative Commons license, unless indicated otherwise in a credit line to the material. If material is not included in the article's Creative Commons license and your intended use is not permitted by statutory regulation or exceeds the permitted use, you will need to obtain permission directly from the copyright holder. To view a copy of this license, visit http:// creativecommons.org/licenses/by/4.0/.

(C) The Author(s) 2019 
Roy Burstein ${ }^{1,612}$, Nathaniel J. Henry ${ }^{1,612}$, Michael L. Collison ${ }^{1}$, Laurie B. Marczak ${ }^{1}$, Amber Sligar ${ }^{1}$, Stefanie Watson ${ }^{1}$, Neal Marquez ${ }^{1}$, Mahdieh Abbasalizad-Farhangi ${ }^{2}$, Masoumeh Abbasi $^{3}$, Foad Abd-Allah ${ }^{4}$, Amir Abdoli ${ }^{5}$, Mohammad Abdollahi ${ }^{6}$, Ibrahim Abdollahpour ${ }^{7,8}$, Rizwan Suliankatchi Abdulkader ${ }^{9}$, Michael R. M. Abrigo ${ }^{10}$, Dilaram Acharya ${ }^{11,12}$, Oladimeji M Adebayo $^{13}$, Victor Adekanmbi ${ }^{14}$, Davoud Adham ${ }^{15}$, Mahdi Afshari ${ }^{16}$, Mohammad Aghaali ${ }^{17}$, Keivan Ahmadi ${ }^{18}$, Mehdi Ahmadi ${ }^{19}$, Ehsan Ahmadpour ${ }^{20}$, Rushdia Ahmed ${ }^{21,22}$, Chalachew Genet Akal $^{23}$, Joshua O. Akinyemi ${ }^{24}$, Fares Alahdab ${ }^{25}$, Noore Alam ${ }^{26}$, Genet Melak Alamene ${ }^{27}$, Kefyalew Addis Alene ${ }^{28,29}$, Mehran Alijanzadeh ${ }^{30}$, Cyrus Alinia $^{31}$, Vahid Alipour ${ }^{32,33}$, Syed Mohamed Aljunid ${ }^{34,35}$, Mohammed J. Almalki ${ }^{36,37}$, Hesham M. Al-Mekhlafi ${ }^{38,39}$, Khalid Altirkawi ${ }^{40}$, Nelson Alvis-Guzman ${ }^{41,42}$, Adeladza Kofi Amegah ${ }^{43}$, Saeed Amini ${ }^{44}$, Arianna Maever Loreche Amit ${ }^{45,46}$, Zohreh Anbari ${ }^{44}$, Sofia Androudi ${ }^{47}$, Mina Anjomshoa ${ }^{48}$, Fereshteh Ansari ${ }^{49}$, Carl Abelardo T. Antonio ${ }^{50,51}$, Jalal Arabloo ${ }^{33}$, Zohreh Arefi ${ }^{52}$, Olatunde Aremu ${ }^{53}$, Bahram Armoon ${ }^{54,55}$, Amit Arora ${ }^{56,57}$, Al Artaman ${ }^{58}$, Anvar Asadi ${ }^{59}$, Mehran Asadi-Aliabadi ${ }^{60}$ Amir Ashraf-Ganjouei ${ }^{7,61}$, Reza Assadi ${ }^{62}$, Bahar Ataeinia ${ }^{63}$, Sachin R. Atre ${ }^{64,65}$, Beatriz Paulina Ayala Quintanilla ${ }^{66,67}$, Martin Amogre Ayanore ${ }^{68}$, Samad Azari ${ }^{33}$, Ebrahim Babaee ${ }^{60}$, Arefeh Babazadeh $^{69}$, Alaa Badawi ${ }^{70,71}$, Soghra Bagheri ${ }^{3}$, Mojtaba Bagherzadeh ${ }^{72}$, Nafiseh Baheiraei $^{73,74}$, Abbas Balouchi ${ }^{75}$, Aleksandra Barac ${ }^{76,77}$, Quique Bassat ${ }^{78,79}$, Bernhard T. Baune $^{80}$, Mohsen Bayati ${ }^{81}$, Neeraj Bedi ${ }^{37,82}$, Ettore Beghi ${ }^{83}$, Masoud Behzadifar ${ }^{84}$, Meysam Behzadifar $^{85}$, Yared Belete Belay ${ }^{86}$, Brent Bell ${ }^{1}$, Michelle L. Bell ${ }^{87}$, Dessalegn Ajema Berbada ${ }^{88}$ Robert S. Bernstein ${ }^{89,90}$, Natalia V. Bhattacharjee ${ }^{1}$, Suraj Bhattarai ${ }^{91,92}$, Zulfiqar A. Bhutta ${ }^{93,94}$, Ali Bijani ${ }^{95}$, Somayeh Bohlouli ${ }^{96}$, Nicholas J. K. Breitborde ${ }^{97,98}$, Gabrielle Britton ${ }^{99,100}$, Annie J. Browne ${ }^{101}$, Sharath Burugina Nagaraja ${ }^{102}$, Reinhard Busse ${ }^{103}$, Zahid A. Butt ${ }^{104,105}$, Josip Car $^{106,107}$, Rosario Cárdenas ${ }^{108}$, Carlos A. Castañeda-Orjuela ${ }^{109,110}$, Ester Cerin ${ }^{111,112}$, Wagaye Fentahun Chanie ${ }^{113}$, Pranab Chatterjee ${ }^{114}$, Dinh-Toi Chu ${ }^{115}$, Cyrus Cooper ${ }^{116,117}$, Vera M. Costa $^{118}$, Koustuv Dalal ${ }^{119,120}$, Lalit Dandona ${ }^{1,121}$, Rakhi Dandona ${ }^{1,121}$, Farah Daoud ${ }^{1}$, Ahmad Daryani $^{122}$, Rajat Das Gupta ${ }^{21}$, Ian Davis ${ }^{1}$, Nicole Davis Weaver ${ }^{1}$, Dragos Virgil Davitoiu ${ }^{123,124}$ Jan-Walter De Neve ${ }^{125}$, Feleke Mekonnen Demeke ${ }^{126}$, Gebre Teklemariam Demoz ${ }^{127,128}$, Kebede Deribe $^{129,130}$, Rupak Desai ${ }^{131}$, Aniruddha Deshpande ${ }^{1}$, Hanna Demelash Desyibelew ${ }^{132}$, Sagnik Dey $^{133}$, Samath Dhamminda Dharmaratne ${ }^{1,134}$, Meghnath Dhimal ${ }^{135}$, Daniel Diaz ${ }^{136,137}$, Leila Doshmangir $^{138}$, Andre R. Duraes ${ }^{139,140}$, Laura Dwyer-Lindgren ${ }^{1,141}$, Lucas Earl ${ }^{1}$, Roya Ebrahimi $^{142}$, Soheil Ebrahimpour ${ }^{69}$, Andem Effiong ${ }^{143}$, Aziz Eftekhari ${ }^{144,145}$, Elham EhsaniChimeh $^{146}$, Iman El Sayed ${ }^{147}$, Maysaa El Sayed Zaki ${ }^{148}$, Maha El Tantawi ${ }^{149,150}$, Ziad ElKhatib $^{151}$, Mohammad Hassan Emamian ${ }^{152}$, Shymaa Enany ${ }^{153}$, Sharareh Eskandarieh ${ }^{7}$, Oghenowede Eyawo ${ }^{154,155}$, Maha Ezalarab ${ }^{1}$, Mahbobeh Faramarzi ${ }^{156}$, Mohammad Fareed ${ }^{157}$, Roghiyeh Faridnia ${ }^{158}$, Andre Faro ${ }^{159}$, Ali Akbar Fazaeli ${ }^{160}$, Mehdi Fazlzadeh ${ }^{161,162}$, Netsanet Fentahun $^{163}$, Seyed-Mohammad Fereshtehnejad ${ }^{164,165}$, João C. Fernandes ${ }^{166}$, Irina Filip ${ }^{167,168}$, Florian Fischer ${ }^{169}$, Nataliya A. Foigt ${ }^{170}$, Masoud Foroutan ${ }^{171}$, Joel Msafiri Francis ${ }^{172}$, Takeshi Fukumoto $^{173,174}$, Nancy Fullman ${ }^{1}$, Silvano Gallus ${ }^{175}$, Destallem Gebremedhin Gebre ${ }^{176,177}$, Tsegaye Tewelde Gebrehiwot ${ }^{178}$, Gebreamlak Gebremedhn Gebremeskel ${ }^{179,180}$, Bradford D. Gessner ${ }^{181,182}$, Birhanu Geta ${ }^{183}$, Peter W. Gething ${ }^{101}$, Reza Ghadimi ${ }^{184}$, Keyghobad Ghadiri ${ }^{3}$, Mahsa Ghajarzadeh ${ }^{185}$, Ahmad Ghashghaee ${ }^{186}$, Paramjit Singh Gill ${ }^{187}$, Tiffany K. Gill ${ }^{188}$, Nick Golding ${ }^{609}$, Nelson G. M. Gomes ${ }^{189,190}$, Philimon N. Gona ${ }^{191}$, Sameer Vali Gopalani ${ }^{192,193}$, Giuseppe Gorini ${ }^{194}$, Bárbara Niegia Garcia Goulart ${ }^{195}$, Nicholas Graetz ${ }^{1}$, Felix Greaves ${ }^{196,197}$, Manfred S. Green ${ }^{198}$, Yuming Guo ${ }^{199,200}$, Arvin Haj-Mirzaian ${ }^{201,202}$, Arya Haj-Mirzaian ${ }^{201,203}$, Brian James Hall ${ }^{204}$, Samer Hamidi ${ }^{205}$, Hamidreza Haririan ${ }^{206}$, Josep Maria Haro ${ }^{207,208}$, Milad Hasankhani ${ }^{209}$, Edris Hasanpoor ${ }^{210}$, Amir Hasanzadeh ${ }^{211,212}$, Hadi Hassankhani ${ }^{213,214}$, Hamid Yimam Hassen ${ }^{215,216}$, Mohamed I. Hegazy ${ }^{4}$, Delia Hendrie ${ }^{217}$, Fatemeh Heydarpour ${ }^{218}$, Thomas R. Hird ${ }^{199,219}$, Chi Linh Hoang ${ }^{220}$, Gillian Hollerich ${ }^{1}$, Enayatollah Homaie Rad ${ }^{221,222}$, Mojtaba Hoseini-Ghahfarokhi ${ }^{223}$, Naznin Hossain ${ }^{22,224}$, Mostafa Hosseini ${ }^{225}$, Mehdi Hosseinzadeh ${ }^{33,226}$, Mihaela Hostiuc ${ }^{123,227}$, Sorin Hostiuc ${ }^{228,229}$, Mowafa Househ ${ }^{230,231}$, Mohamed Hsairi ${ }^{232}$, Olayinka Stephen Ilesanmi ${ }^{233}$, Mohammad Hasan Imani-Nasab ${ }^{234}$, Usman Iqbal ${ }^{235}$, Seyed Sina Naghibi Irvani ${ }^{236}$, Nazrul Islam ${ }^{237,238}$, Sheikh Mohammed Shariful Islam ${ }^{239,240}$, Mikk Jürisson ${ }^{241}$, Nader Jafari Balalami ${ }^{242}$, Amir Jalalii ${ }^{24}$, Javad Javidnia ${ }^{244}$, Achala Upendra Jayatilleke ${ }^{245,246}$, Ensiyeh Jenabi ${ }^{247}$, John S. Ji ${ }^{248}$, Yash B. Jobanputra ${ }^{249}$, Kimberly Johnson ${ }^{1}$, Jost B. Jonas ${ }^{250,251}$, Zahra Jorjoran Shushtari ${ }^{252}$, Jacek Jerzy Jozwiak ${ }^{253,254}$, Ali Kabir ${ }^{255}$, Amaha Kahsay $^{256}$, Hamed Kalani ${ }^{257,258}$, Rohollah Kalhor ${ }^{259}$, Manoochehr Karami ${ }^{260}$, Surendra Karki $^{261,262}$, Amir Kasaeian ${ }^{263,264}$, Nicholas J. Kassebaum ${ }^{1,265}$, Peter Njenga Keiyoro ${ }^{266}$, Grant Rodgers Kemp ${ }^{1,267}$, Roghayeh Khabiri ${ }^{268,269}$, Yousef Saleh Khader $^{270}$, Morteza Abdullatif Khafaie $^{271}$, Ejaz Ahmad Khan ${ }^{272}$, Junaid Khan ${ }^{273}$, Muhammad Shahzeb Khan ${ }^{274,275}$, Young-Ho Khang $^{276,277}$, Khaled Khatab ${ }^{278,279}$, Amir Khater ${ }^{280}$, Mona M. Khater ${ }^{281}$, Alireza Khatony ${ }^{3}$, Mohammad Khazaei ${ }^{282}$, Salman Khazaei ${ }^{260}$, Maryam Khazaei-Pool ${ }^{283}$, Jagdish Khubchandani ${ }^{284}$, Neda Kianipour ${ }^{3,285}$, Yun Jin Kim ${ }^{286}$, Ruth W. Kimokoti ${ }^{287}$, Damaris K. Kinyoki $^{1,141}$, Adnan Kisa ${ }^{288,289}$, Sezer Kisa ${ }^{290}$, Tufa Kolola ${ }^{291}$, Soewarta Kosen ${ }^{292}$, Parvaiz A. Koull $^{293}$, Ai Koyanagi ${ }^{79,294}$, Moritz U. G. Kraemer ${ }^{295,296}$, Kewal Krishan ${ }^{297}$, Kris J. Krohn', Nuworza Kugbey ${ }^{298,299}$, G. Anil Kumar' ${ }^{121}$, Manasi Kumar ${ }^{300,301}$, Pushpendra Kumar ${ }^{302}$, Desmond Kuupiel ${ }^{303,304}$, Ben Lacey ${ }^{305,306}$, Sheetal D. Lad ${ }^{307}$, Faris Hasan Lami ${ }^{308}$, Anders O. Larsson $^{309,310}$, Paul H. Lee ${ }^{311}$, Mostafa Leili ${ }^{282}$, Aubrey J. Levine ${ }^{1}$, Shanshan $\mathrm{Li}^{199}$, Lee-Ling Lim $^{312,313}$, Stefan Listl ${ }^{314,315}$, Joshua Longbottom ${ }^{316}$, Jaifred Christian F. Lopez ${ }^{45,317}$, Stefan Lorkowski ${ }^{318,319}$, Sameh Magdeldin ${ }^{320,321}$, Hassan Magdy Abd El Razek ${ }^{322}$, Muhammed Magdy Abd El Razek ${ }^{323}$, Azeem Majeed ${ }^{196}$, Afshin Maleki ${ }^{142}$, Reza Malekzadeh ${ }^{324,325}$, Deborah Carvalho Malta $^{326}$, Abdullah A. Mamun ${ }^{327}$, Navid Manafi ${ }^{328,329}$, Ana-Laura Manda ${ }^{330}$, Morteza Mansourian $^{331}$, Francisco Rogerlândio Martins-Melo ${ }^{332}$, Anthony Masaka ${ }^{333}$, Benjamin Ballard Massenburg $^{334}$, Pallab K. Maulik ${ }^{335,336}$, Benjamin K. Mayala ${ }^{1}$, Mohsen Mazidi ${ }^{337}$, Martin McKee $^{338}$, Ravi Mehrotra ${ }^{339}$, Kala M. Mehta ${ }^{340}$, Gebrekiros Gebremichael Meles ${ }^{88}$, Walter Mendoza $^{341}$, Ritesh G. Menezes ${ }^{342}$, Atte Meretoja ${ }^{343,344}$, Tuomo J. Meretoja ${ }^{345,346}$, Tomislav Mestrovic $^{347,348}$, Ted R. Miller ${ }^{217,349}$, Molly K. Miller-Petrie ${ }^{1}$, Edward J. Mills ${ }^{350}$, George J. Milne $^{351}$, G. K. Mini ${ }^{352}$, Seyed Mostafa Mir ${ }^{353,354}$, Hamed Mirjalali ${ }^{355}$, Erkin M. Mirrakhimov $^{356,357}$, Efat Mohamadi ${ }^{358}$, Dara K. Mohammad ${ }^{359,360}$, Aso Mohammad Darwesh $^{361}$, Naser Mohammad Gholi Mezerji ${ }^{362}$, Ammas Siraj Mohammed ${ }^{363}$, Shafiu Mohammed $^{364,365}$, Ali H. Mokdad ${ }^{1,141}$, Mariam Molokhia ${ }^{366}$, Lorenzo Monasta ${ }^{367}$, Yoshan Moodley $^{303}$, Mahmood Moosazadeh ${ }^{368}$, Ghobad Moradi ${ }^{369,370}$, Masoud Moradi ${ }^{3,59}$, Yousef Moradi $^{371}$, Maziar Moradi-Lakeh ${ }^{60}$, Mehdi Moradinazar ${ }^{59}$, Paula Moraga ${ }^{372}$, Lidia Morawska ${ }^{373}$, Abbas Mosapour ${ }^{353,374}$, Seyyed Meysam Mousavi ${ }^{375}$, Ulrich Otto Mueller ${ }^{376,377}$, Atalay Goshu
Muluneh $^{378}$, Ghulam Mustafa ${ }^{379,380}$, Behnam Nabavizadeh ${ }^{381}$, Mehdi Naderi ${ }^{382}$, Ahamarshan Jayaraman Nagarajan ${ }^{383,384}$, Azin Nahvijou ${ }^{385}$, Farid Najafi ${ }^{386}$, Vinay Nangia ${ }^{387}$, Duduzile Edith Ndwandwe ${ }^{388}$, Nahid Neamati ${ }^{353}$, Ionut Negoi ${ }^{389,390}$, Ruxandra Irina Negoi ${ }^{391,392}$, Josephine W. Ngunjiri ${ }^{393}$, Huong Lan Thi Nguyen ${ }^{394}$, Long Hoang Nguyen ${ }^{395}$, Son Hoang Nguyen ${ }^{395}$, Katie R Nielsen ${ }^{396,397}$, Dina Nur Anggraini Ningrum ${ }^{610,611}$, Yirga Legesse Nirayo ${ }^{398}$, Molly R. Nixon ${ }^{1}$, Chukwudi A. Nnaji ${ }^{388,399}$, Marzieh Nojomi ${ }^{60,400}$, Mehdi Noroozi ${ }^{401}$, Shirin Nosratnejad ${ }^{402}$, Jean Jacques Noubiap ${ }^{403}$, Soraya Nouraei Motlagh ${ }^{234}$, Richard Ofori-Asenso ${ }^{404,405}$, Felix Akpojene Ogbo $^{406}$, Kelechi E. Oladimeji ${ }^{303,407}$, Andrew T. Olagunju ${ }^{408,409}$, Meysam Olfatifar ${ }^{410}$, Solomon Olum $^{411,412}$, Bolajoko Olubukunola Olusanya ${ }^{413}$, Mojisola Morenike Oluwasanu ${ }^{414}$, Obinna E. Onwujekwe $^{415}$, Eyal Oren ${ }^{416,417}$, Doris D. V. Ortega-Altamirano ${ }^{418}$, Alberto Ortiz ${ }^{419,420}$, Osayomwanbo Osarenotor ${ }^{421}$, Frank B. Osei ${ }^{422,423}$, Aaron E. Osgood-Zimmerman ${ }^{1}$, Stanislav S. Otstavnov ${ }^{424,425}$, Mayowa Ojo Owolabi ${ }^{426}$, Mahesh P. A. ${ }^{427}$, Abdol Sattar Pagheh ${ }^{122}$, Smita Pakhale ${ }^{428}$, Songhomitra Panda-Jonas ${ }^{429}$, Animika Pandey ${ }^{121}$, Eun-Kee Park ${ }^{430}$, Hadi Parsian $^{353}$, Tahereh Pashaei ${ }^{142}$, Sangram Kishor Patel ${ }^{431,432}$, Veincent Christian Filipino Pepito $^{433}$, Alexandre Pereira ${ }^{434,435}$, Samantha Perkins ${ }^{1}$, Brandon V. Pickering ${ }^{1}$, Thomas Pilgrim $^{436}$, Majid Pirestani ${ }^{437}$, Bakhtiar Piroozi ${ }^{370}$, Meghdad Pirsaheb ${ }^{3}$, Oleguer PlanaRipoll $^{438}$, Hadi Pourjafar ${ }^{439,440}$, Parul Puri ${ }^{302}$, Mostafa Qorbani ${ }^{441}$, Hedley Quintana ${ }^{100}$, Mohammad Rabiee ${ }^{442}$, Navid Rabiee ${ }^{72}$, Amir Radfar ${ }^{443,444}$, Alireza Rafiei ${ }^{445,446}$, Fakher Rahim $^{447,448}$, Zohreh Rahimi ${ }^{449}$, Vafa Rahimi-Movaghar ${ }^{450}$, Shadi Rahimzadeh ${ }^{63}$, Fatemeh Rajati $^{451}$, Sree Bhushan Raju ${ }^{452}$, Azra Ramezankhani ${ }^{453,454}$, Chhabi Lal Ranabhat ${ }^{455,456}$, Davide Rasella $^{457,458}$, Vahid Rashedi ${ }^{459}$, Lal Rawal ${ }^{56,460}$, Robert C. Reiner Jr ${ }^{1,141}$, Andre M. N. Renzaho $^{461}$, Satar Rezaei ${ }^{462}$, Aziz Rezapour ${ }^{33}$, Seyed Mohammad Riahi ${ }^{463,464}$, Ana Isabel Ribeiro $^{465}$, Leonardo Roever ${ }^{466}$, Elias Merdassa Roro ${ }^{467,468}$, Max Roser ${ }^{469}$, Gholamreza Roshandel $^{324,470}$, Daem Roshani ${ }^{471}$, Ali Rostami ${ }^{472}$, Enrico Rubagotti ${ }^{473,474}$, Salvatore Rubino $^{475}$, Siamak Sabour ${ }^{463}$, Nafis Sadat ${ }^{1}$, Ehsan Sadeghi ${ }^{59}$, Reza Saeedi ${ }^{476}$, Yahya Safari ${ }^{3}$, Roya Safari-Faramani ${ }^{477}$, Mahdi Safdarian ${ }^{450,478}$, Amirhossein Sahebkar ${ }^{479,480}$, Mohammad Reza Salahshoor ${ }^{481}$, Nasir Salam ${ }^{482}$, Payman Salamati ${ }^{450,483}$, Farkhonde Salehi ${ }^{484}$, Saleh Salehi Zahabi $^{485,486}$, Yahya Salimi ${ }^{386}$, Hamideh Salimzadeh ${ }^{324}$, Joshua A. Salomon ${ }^{487}$, Evanson Zondani Sambala ${ }^{388}$, Abdallah M. Samy ${ }^{488}$, Milena M. Santric Milicevic ${ }^{489}$, Bruno Piassi Sao Jose $^{490}$, Sivan Yegnanarayana Iyer Saraswathy ${ }^{491,492}$, Rodrigo Sarmiento-Suárez ${ }^{493}$, Benn Sartorius $^{141,494}$, Brijesh Sathian ${ }^{495,496}$, Sonia Saxena ${ }^{497}$, Alyssa N. Sbarra ${ }^{1}$, Lauren E. Schaeffer ${ }^{1}$, David C. Schwebel ${ }^{498}$, Sadaf G. Sepanlou ${ }^{324,325}$, Seyedmojtaba Seyedmousavi ${ }^{499,500}$, Faramarz Shaahmadi $^{501}$, Masood Ali Shaikh ${ }^{502}$, Mehran Shams-Beyranvand ${ }^{63,503}$, Amir Shamshirian ${ }^{504}$, Morteza Shamsizadeh $^{505}$, Kiomars Sharafi ${ }^{3}$, Mehdi Sharif ${ }^{506,507}$, Mahdi Sharif-Alhoseini ${ }^{450}$, Hamid Sharifi ${ }^{508}$, Jayendra Sharma ${ }^{509}$, Rajesh Sharma ${ }^{510}$, Aziz Sheikh ${ }^{511,512}$, Chloe Shields ${ }^{1}$, Mika Shigematsu ${ }^{513}$, Rahman Shiri ${ }^{514}$, Ivy Shiue ${ }^{515}$, Kerem Shuval ${ }^{198}$, Tariq J. Siddiqi ${ }^{275}$, João Pedro Silva ${ }^{118}$, Jasvinder A. Singh ${ }^{516,517}$, Dhirendra Narain Sinha ${ }^{518,519}$, Malede Mequanent Sisay $^{378,520}$, Solomon Sisay ${ }^{521}$, Karen Sliwa ${ }^{403}$, David L. Smith ${ }^{1,141}$, Ranjani Somayaji ${ }^{522,523}$, Moslem Soofi ${ }^{524}$, Joan B. Soriano ${ }^{525,526}$, Chandrashekhar T. Sreeramareddy ${ }^{527}$, Agus Sudaryanto ${ }^{528}$, Mu'awiyyah Babale Sufiyan ${ }^{529}$, Bryan L. Sykes $^{530}$, P. N. Sylaja ${ }^{531}$, Rafael Tabarés-Seisdedos ${ }^{532,533}$, Karen M. Tabb ${ }^{534}$, Takahiro Tabuchi ${ }^{535}$, Nuno Taveira ${ }^{536,537}$, Mohamad-Hani Temsah ${ }^{538,539}$, Abdullah Sulieman Terkawi ${ }^{540,541}$, Zemenu Tadesse Tessema ${ }^{378}$, Kavumpurathu Raman Thankappan ${ }^{542}$, Sathish Thirunavukkarasu ${ }^{543}$, Quyen G. To ${ }^{544}$, Marcos Roberto Tovani-Palone ${ }^{545}$, Bach Xuan Tran ${ }^{546}$, Khanh Bao Tran ${ }^{547,548}$, Irfan Ullah ${ }^{549,550}$, Muhammad Shariq Usman ${ }^{275}$, Olalekan A. Uthman ${ }^{551}$, Amir Vahedian-Azimi ${ }^{552,553}$, Pascual R. Valdez $^{554,555}$, Job F. M. van Boven ${ }^{556,557}$, Tommi Juhani Vasankari ${ }^{558}$, Yasser Vasseghian ${ }^{462}$, Yousef Veisani $^{559}$, Narayanaswamy Venketasubramanian ${ }^{560,561}$, Francesco S. Violante ${ }^{562,563}$, Sergey Konstantinovitch Vladimirov ${ }^{564,565}$, Vasily Vlassov ${ }^{566}$, Theo $\operatorname{Vos}^{1,141}$, Giang Thu Vu' ${ }^{220}$ Isidora S. Vujcic ${ }^{77}$, Yasir Waheed ${ }^{567}$, Jon Wakefield ${ }^{568}$, Haidong Wang ${ }^{1,141}$, Yafeng Wang ${ }^{569}$, Yuan-Pang Wang ${ }^{570}$, Joseph L. Ward ${ }^{571}$, Robert G. Weintraub ${ }^{572,573}$, Kidu Gidey Weldegwergs $^{398}$, Girmay Teklay Weldesamuel ${ }^{574}$, Ronny Westerman ${ }^{575}$, Charles Shey Wiysonge $^{576,577}$, Dawit Zewdu Wondafrash ${ }^{578,579}$, Lauren Woyczynski ${ }^{1}, \mathrm{Ai}^{-}-\mathrm{Min} \mathrm{Wu}^{580}$, Gelin $\mathrm{Xu}^{581}$, Abbas Yadegar ${ }^{355}$, Tomohide Yamada ${ }^{582}$, Vahid Yazdi-Feyzabadi ${ }^{583,584}$, Christopher Sabo Yilgwan ${ }^{585,586}$, Paul Yip ${ }^{587,588}$, Naohiro Yonemoto ${ }^{589}$, Javad Yoosefi Lebni ${ }^{331}$, Mustafa Z. Younis ${ }^{590,591}$, Mahmoud Yousefifard ${ }^{592}$, Hebat-Allah Salah A. Yousof ${ }^{593}$ Chuanhua Yu ${ }^{569,594}$, Hasan Yusefzadeh ${ }^{595}$, Erfan Zabeh ${ }^{596,597}$, Telma Zahirian Moghadam ${ }^{33,598}$ Sojib Bin Zaman ${ }^{599,600}$, Mohammad Zamani ${ }^{601}$, Hamed Zandian ${ }^{598,602}$, Alireza Zangeneh ${ }^{603}$, Taddese Alemu Zerfu ${ }^{604,605}$, Yunquan Zhang ${ }^{606,607}$, Arash Ziapour ${ }^{331}$, Sanjay Zodpey ${ }^{608}$, Christopher J. L. Murray ${ }^{1,141,613}$ \& Simon I. Hay ${ }^{1,141,613 *}$

Institute for Health Metrics and Evaluation, University of Washington, Seattle, WA, USA. ${ }^{2}$ Department of Nutrition, Tabriz University of Medical Sciences, Tabriz, Iran. ${ }^{3}$ Kermanshah University of Medical Sciences, Kermanshah, Iran. ${ }^{4}$ Department of Neurology, Cairo University, Cairo, Egypt. ${ }^{5}$ Department of Parasitology and Mycology, Jahrom University of Medical Sciences, Jahrom, Iran. ${ }^{6}$ The Institute of Pharmaceutical Sciences, Tehran University of Medical Sciences, Tehran, Iran. ${ }^{7}$ Multiple Sclerosis Research Center, Tehran University of Medical Sciences, Tehran, Iran. ${ }^{8}$ Department of Epidemiology, Arak University of Medical Sciences, Arak, Iran. ${ }^{9}$ Department of Public Health, Ministry of Health, Riyadh, Saudi Arabia. ${ }^{10}$ Research Department, Philippine Institute for Development Studies, Quezon City, The Philippines. ${ }^{11}$ Department of Preventive Medicine, Dongguk University, Gyeongju, South Korea. ${ }^{12}$ Department of Community Medicine, Kathmandu University, Devdaha, Nepal. ${ }^{13}$ College of Medicine, University College Hospital, Ibadan, Nigeria. ${ }^{14}$ School of Medicine, Cardiff University, Cardiff, UK. ${ }^{15}$ School of Health, Ardabil University of Medical Science, Ardabil, Iran. ${ }^{16}$ Department of Community Medicine, Zabol University of Medical Sciences, Zabol, Iran. ${ }^{17}$ Department of Epidemiology and Biostatistics, Qom University of Medical Sciences, Qom, Iran. ${ }^{18}$ School of Pharmacy, University of Lincoln, Lincoln, UK. ${ }^{19}$ Environmental Technologies Research Center, Ahvaz Jundishapur University of Medical Sciences, Ahvaz, Iran. ${ }^{20}$ Department of Parasitology and Mycology, Tabriz University of Medical Sciences, Tabriz, Iran. ${ }^{21}$ James P. Grant School of Public Health, Brac University, Dhaka, Bangladesh. ${ }^{22}$ Health Systems and Population Studies Division, International Centre for Diarrhoeal Disease Research Bangladesh, Dhaka, Bangladesh. ${ }^{23}$ Department of Medical Laboratory Science, Bahir Dar University, Bahir 
RESEARCH ARTICLE

Dar, Ethiopia. ${ }^{24}$ Epidemiology and Medical Statistics, University of Ibadan, Ibadan, Nigeria. ${ }^{25}$ Evidence Based Practice Center, Mayo Clinic Foundation for Medical Education and Research, Rochester, MN, USA. ${ }^{26}$ Prevention Division, Queensland Health, Herston, Queensland, Australia. ${ }^{27}$ School of Health Sciences, Madda Walabu University, Bale Goba, Ethiopia. ${ }^{28}$ Institute of Public Health, University of Gondar, Gondar, Ethiopia. ${ }^{29}$ Research School of Population Health, Australian National University, Canberra, Australian Capital Territory, Australia. ${ }^{30}$ Qazvin University of Medical Sciences, Qazvin, Iran. ${ }^{31}$ Department of Health Care Management and Economics, Urmia University of Medical Science, Urmia, Iran. ${ }^{32}$ Health Economics Department Iran University of Medical Sciences, Tehran, Iran. ${ }^{33} \mathrm{Health}$ Management and Economics Research Center, Iran University of Medical Sciences, Tehran, Iran. ${ }^{34}$ Department of Health Policy and Management, Kuwait University, Safat, Kuwait. ${ }^{35}$ International Centre for Casemix and Clinical Coding, National University of Malaysia, Bandar Tun Razak, Malaysia. ${ }^{36}$ Faculty of Public Health and Tropical Medicine, Jazan University, Jazan, Saudi Arabia. ${ }^{37}$ Jazan University, Jazan, Saudi Arabia. ${ }^{38}$ Medical Research Center, Jazan University, Jazan, Saudi Arabia. ${ }^{39}$ Department of Medical Parasitology, Sana'a University, Sana'a, Yemen. ${ }^{40}$ King Saud University, Riyadh, Saudi Arabia. ${ }^{41}$ Research Group in Health Economics, Universidad de Cartagena, Cartagena, Colombia. ${ }^{42}$ Research Group in Hospital Management and Health Policies, Universidad de la Costa, Barranquilla, Colombia. ${ }^{43}$ Biomedical Science, University of Cape Coast, Cape Coast, Ghana. ${ }^{44}$ Health Services Management Department, Arak University of Medical Sciences, Arak, Iran. ${ }^{45}$ Department of Epidemiology and Biostatistics, University of the Philippines Manila, Manila, The Philippines. ${ }^{46}$ Online Programs for Applied Learning, Johns Hopkins University, Baltimore, MD, USA. ${ }^{47}$ Department of Medicine, University of Thessaly, Volos, Greece. ${ }^{48}$ Social Determinants of Health Research Center, Rafsanjan University of Medical Sciences, Rafsanjan, Iran. ${ }^{49}$ Research Center for Evidence Based Medicine-Health Management and Safety Promotion Research Institute, Tabriz University of Medical Sciences, Tabriz, Iran. ${ }^{50}$ Department of Health Policy and Administration, University of the Philippines Manila, Manila, The Philippines. ${ }^{51}$ Department of Applied Social Sciences, Hong Kong Polytechnic University, Hong Kong, China. ${ }^{52}$ Department of Health Promotion and Education, Tehran University of Medical Sciences, Tehran, Iran. ${ }^{53}$ School of Health Sciences, Birmingham City University, Birmingham, UK. ${ }^{54}$ School of Nursing and Midwifery, Saveh University of Medical Sciences, Saveh, Iran. ${ }^{55}$ Social Determinants of Health Research Center, Saveh University of Medical Sciences, Saveh, Iran. ${ }^{56}$ School of Science and Health, Western Sydney University, Penrith, New South Wales, Australia. ${ }^{57}$ Oral Health Services, Sydney Local Health District, Sydney, New South Wales, Australia. ${ }^{58}$ Department of Community Health Sciences, University of Manitoba, Winnipeg, Manitoba, Canada. ${ }^{59}$ Research Center for Environmental Determinants of Health, Kermanshah University of Medical Sciences, Kermanshah, Iran. ${ }^{60}$ Preventive Medicine and Public Health Research Center, Iran University of Medical Sciences, Tehran, Iran. ${ }^{61}$ Faculty of Medicine, Tehran University of Medical Sciences, Tehran, Iran. ${ }^{62}$ Education Development Center, Mashhad University of Medical Sciences, Mashhad, Iran. ${ }^{63}$ Non-communicable Diseases Research Center, Tehran University of Medical Sciences, Tehran, Iran. ${ }^{64}$ Center for Clinical Global Health Education, Johns Hopkins University, Baltimore, MD, USA. ${ }^{65} \mathrm{Dr}$ D. Y. Patil Medical College, Pune, India. ${ }^{66}$ The Judith Lumley Centre, La Trobe University, Melbourne, Victoria, Australia. ${ }^{67}$ General Office for Research and Technological Transfer, Peruvian National Institute of Health, Lima, Peru. ${ }^{68}$ Department of Family and Community Health, University of Health and Allied Sciences, Ho, Ghana. ${ }^{69} \mathrm{Center}$ for Infectious Diseases Research, Babol, Iran. ${ }^{70}$ Public Health Risk Sciences Division, Public Health Agency of Canada, Toronto, Ontario, Canada. ${ }^{71}$ Department of Nutritional Sciences, University of Toronto, Toronto, Ontario, Canada. ${ }^{72}$ Department of Chemistry, Sharif University of Technology, Tehran, Iran. ${ }^{73}$ Tissue Engineering and Applied Cell Sciences Division, Tarbiat Modares University, Tehran, Iran. ${ }^{74}$ Division of Diseases, Advanced Technologies Research Group, Tehran, Iran. ${ }^{75}$ School of Nursing and Midwifery, Iran University of Medical Sciences, Tehran, Iran. ${ }^{76}$ Clinic for Infectious and Tropical Diseases, Clinical Center of Serbia, Belgrade, Serbia. ${ }^{77}$ Faculty of Medicine, University of Belgrade, Belgrade, Serbia. ${ }^{78}$ Barcelona Institute for Global Health, University of Barcelona, Barcelona, Spain. ${ }^{79}$ Catalan Institution for Research and Advanced Studies (ICREA), Barcelona, Spain. ${ }^{80}$ Department of Psychiatry, Melbourne Medical School, Melbourne, Victoria, Australia. ${ }^{81}$ Health Human Resources Research Center, Shiraz University of Medical Sciences, Shiraz, Iran. ${ }^{82}$ Department of Community Medicine, Gandhi Medical College Bhopal, Bhopal, India. ${ }^{83}$ Department of Neuroscience, Mario Negri Institute for Pharmacological Research, Milan, Italy. ${ }^{84}$ Social Determinants of Health Research Center, Lorestan University of Medical Sciences, Khorramabad, Iran. ${ }^{85} \mathrm{Hepatitis}$ Research Center, Lorestan University of Medical Sciences, Khorramabad, Iran. ${ }^{86}$ Pharmacoepidemiology and Social Pharmacy, Mekelle University, Mekelle, Ethiopia. ${ }^{87}$ School of Forestry and Environmental Studies, Yale University, New Haven, CT, USA. ${ }^{88}$ Department of Public Health, Arba Minch University, Arba Minch, Ethiopia. ${ }^{89}$ Hubert Department of Global Health, Emory University, Atlanta, GA, USA. ${ }^{90}$ Department of Global Health, University of South Florida, Tampa, FL, USA. ${ }^{91}$ London School of Hygiene \& Tropical Medicine, London, UK. ${ }^{92} \mathrm{Nepal}$ Academy of Science \& Technology, Patan, Nepal. ${ }^{93}$ The Centre for Global Child Health, Hospital for Sick Children, University of Toronto, Toronto, Ontario, Canada. ${ }^{94}$ Center of Excellence in Women and Child Health, Aga Khan University, Karachi, Pakistan. ${ }^{95}$ Social Determinants of Health Research Center, Babol University of Medical Sciences, Babol, Iran. ${ }^{96}$ Department of Veterinary Medicine, Karaj Islamic Azad University, Kermanshah, Iran. ${ }^{97}$ Department of Psychology, Ohio State University, Columbus, $\mathrm{OH}$, USA. ${ }^{98}$ Psychiatry and Behavioral Health Department, Ohio State University, Columbus, $\mathrm{OH}$ USA. ${ }^{99}$ Neuroscience Department, Institute for Scientific Research and High Technology Services, City of Knowledge, Panama. ${ }^{100}$ Gorgas Memorial Institute for Health Studies, Panama, Panama. ${ }^{101}$ Big Data Institute, Li Ka Shing Centre for Health Information and Discovery, University of Oxford, Oxford, UK. ${ }^{102}$ Department of Community Medicine, Employees' State Insurance Model Hospital, Bangalore, India. ${ }^{103}$ Department for Health Care Management, Technical University of Berlin, Berlin, Germany. ${ }^{104}$ School of Population and Public Health, University of British Columbia, Vancouver, British Columbia, Canada. ${ }^{105} \mathrm{Al}$ Shifa School of Public Health, Al Shifa Trust Eye Hospital, Rawal pindi, Pakistan. ${ }^{106}$ Centre for Population Health Sciences, Nanyang Technological University, Singapore, Singapore. ${ }^{107}$ Global Ehealth Unit, Imperial College London, London, UK. ${ }^{108}$ Department of Population and Health, Metropolitan Autonomous University, Mexico City, Mexico. ${ }^{109}$ Colombian National Health Observatory,
National Institute of Health, Bogota, Colombia. ${ }^{110}$ Epidemiology and Public Health Evaluation Group, National University of Colombia, Bogota, Colombia. ${ }^{111}$ Mary Mackillop Institute for Health Research, Australian Catholic University, Melbourne, Victoria, Australia. ${ }^{112}$ School of Public Health, University of Hong Kong, Hong Kong, China. ${ }^{113}$ Department of Obstetrics and Gynaecology, University of Gondar, Gondar, Ethiopia. ${ }^{114}$ Division of Epidemiology, National Institute of Cholera and Enteric Diseases, Kolkata, India. ${ }^{115}$ Faculty of Biology, Hanoi National University of Education, Hanoi, Vietnam. ${ }^{116}$ Department of Rheumatology, University of Oxford, Oxford, UK. ${ }^{117}$ Medical Research Council Lifecourse Epidemiology Unit, University of Southampton, Southampton, UK. ${ }^{118}$ Applied Molecular Biosciences Unit (UCIBIO), University of Porto, Porto, Portugal. ${ }^{119}$ Institute of Public Health Kalyani, Kalyani, India. ${ }^{120}$ School of Health Science, Orebro University, Orebro, Sweden. ${ }^{121}$ Public Health Foundation of India, Gurugram, India. ${ }^{122}$ Toxoplasmosis Research Center, Mazandaran University of Medical Sciences, Sari, Iran. ${ }^{123}$ Department of General Surgery, Carol Davila University of Medicine and Pharmacy, Bucharest, Romania. ${ }^{124}$ Department of Surgery, Clinical Emergency Hospital St Pantelimon, Bucharest, Romania. ${ }^{125}$ Heidelberg Institute of Global Health (HIGH), Heidelberg University, Heidelberg, Germany. ${ }^{126}$ Bahir Dar University, Bahir Dar, Ethiopia. ${ }^{127}$ School of Pharmacy, Aksum University, Aksum, Ethiopia. ${ }^{128}$ Addis Ababa University, Addis Ababa, Ethiopia. ${ }^{129}$ School of Public Health, Addis Ababa University, Addis Ababa, Ethiopia. ${ }^{130}$ Department of Global Health and Infection, Brighton and Sussex Medical School, Brighton, UK. ${ }^{131}$ Division of Cardiology, Atlanta Veterans Affairs Medical Center, Decatur, GA, USA. ${ }^{132}$ Public Health Nutrition, Bahir Dar University, Bahir Dar, Ethiopia. ${ }^{133}$ Centre for Atmospheric Sciences, Indian Institute of Technology Delhi, New Delhi, India. ${ }^{134}$ Department of Community Medicine, University of Peradeniya, Peradeniya, Sri Lanka. ${ }^{135}$ Health Research Section, Nepal Health Research Council, Kathmandu, Nepal. ${ }^{136}$ Center of Complexity Sciences, National Autonomous University of Mexico, Mexico City, Mexico. ${ }^{137}$ Facultad de Medicina Veterinaria y Zootecnia, Autonomous University of Sinaloa, Culiacan Rosales, Mexico. ${ }^{138}$ Department of Health Policy and Economy, Tabriz University of Medical Sciences, Tabriz, Iran. ${ }^{139}$ School of Medicine, Federal University of Bahia, Salvador, Brazil. ${ }^{140}$ Diretoria Médica, Roberto Santos General Hospital, Salvador, Brazil. ${ }^{141}$ Department of Health Metrics Sciences, School of Medicine, University of Washington, Seattle, WA, USA. ${ }^{142}$ Environmental Health Research Center, Kurdistan University of Medical Sciences, Sanandaj, Iran. ${ }^{143}$ Clinical Epidemiology and Biostatistics, University of Newcastle, Newcastle, New South Wales, Australia. ${ }^{144}$ Department of Toxicology and Pharmacology, Tabriz University of Medical Sciences, Tabriz, Iran. ${ }^{145}$ Department of Basic Sciences, Maragheh University of Medical Sciences, Maragheh, Iran. ${ }^{146}$ National Institute for Health Researchers, Tehran University of Medical Sciences, Tehran, Iran. ${ }^{147}$ Medical Research Institute, Alexandria University, Alexandria, Egypt. ${ }^{148}$ Department of Clinical Pathology, Mansoura University, Mansoura, Egypt. ${ }^{149}$ Pediatric Dentistry and Dental Public Health, Alexandria University, Alexandria, Egypt. ${ }^{150}$ Preventive Dental Sciences, Imam Abdulrahman Bin Faisal University, Dammam, Saudi Arabia. ${ }^{151}$ Department of Public Health Sciences, Karolinska Institutet, Stockholm, Sweden. ${ }^{152}$ Ophthalmic Epidemiology Research Center, Shahroud University of Medical Sciences, Shahroud, Iran. ${ }^{153}$ Department of Microbiology and Immunology, Suez Canal University, Ismailia, Egypt. ${ }^{154}$ Epidemiology and Population Health, British Columbia Centre for Excellence in HIV/AIDS, Vancouver, British Columbia, Canada. ${ }^{155}$ Faculty of Health Sciences, Simon Fraser University, Burnaby, British Columbia, Canada ${ }^{156}$ Babol University of Medical Sciences, Babol, Iran. ${ }^{157}$ College of Medicine, Imam Muhammad Ibn Saud Islamic University, Riyadh, Saudi Arabia. ${ }^{158}$ Department of Parasitology, Mazandaran University of Medical Sciences, Sari, Iran. ${ }^{159}$ Department of Psychology, Federal University of Sergipe, Sao Cristovao, Brazil. ${ }^{160}$ Social Determinants of Health Research Center, Hamadan University of Medical Sciences, Hamadan, Iran. ${ }^{161}$ Environmental Health Engineering, Tehran University of Medical Sciences, Tehran, Iran. ${ }^{162}$ Department of Environmental Health Engineering, Ardabil University of Medical Science, Ardabil, Iran. ${ }^{163}$ Department of Public Health Nutrition, Bahir Dar University, Bahir Dar, Ethiopia. ${ }^{164}$ Department of Neurobiology, Karolinska Institutet, Stockholm, Sweden. ${ }^{165}$ Division of Neurology, University of Ottawa, Ottawa, Ontario, Canada. ${ }^{166}$ Center for Biotechnology and Fine Chemistry, Catholic University of Portugal, Porto, Portugal. ${ }^{167}$ Psychiatry Department, Kaiser Permanente, Fontana, CA, USA. ${ }^{168}$ Department of Health Sciences, A.T. Still University, Mesa, AZ, USA. ${ }^{169}$ Department of Public Health Medicine, Bielefeld University, Bielefeld, Germany. ${ }^{170}$ Institute of Gerontology, National Academy of Medical Sciences of Ukraine, Kyiv, Ukraine. ${ }^{171}$ Abadan School of Medical Sciences, Abadan, Iran. ${ }^{172} \mathrm{Clinical}$ Medicine and Wits Reproductive Health and HIV Institute, University of the Witwatersrand, Johannesburg, South Africa. ${ }^{173}$ Gene Expression \& Regulation Program, Cancer Institute (W.I.A.), Philadelphia, PA, USA. ${ }^{174}$ Department of Dermatology, Kobe University, Kobe, Japan. ${ }^{175}$ Department of Environmental Health Science, Mario Negri Institute for Pharmacological Research, Milan, Italy. ${ }^{176}$ Mekelle University, Mekelle, Ethiopia. ${ }^{177} \mathrm{Dr}$ Tewelde Legesse Health Sciences College, Mekelle, Ethiopia. ${ }^{178}$ Department of Epidemiology, Jimma University, Jimma, Ethiopia. ${ }^{179}$ School of Nursing, Mekelle University, Mekelle, Ethiopia. ${ }^{180}$ Nursing Department, Aksum University, Aksum, Ethiopia. ${ }^{181}$ Vaccines Department, Pfizer, Collegeville, PA, USA. ${ }^{182}$ Agency of Preventive Medicine, Paris, France. ${ }^{183}$ Department of Pharmacy, Wollo University, Dessie, Ethiopia. ${ }^{184} \mathrm{Health}$ Research Institute, Babol University of Medical Sciences, Babol, Iran. ${ }^{185}$ Department of Neurology, Tehran University of Medical Sciences, Tehran, Iran. ${ }^{186}$ Department of Health Services Management, Iran University of Medical Sciences, Tehran, Iran. ${ }^{187}$ Unit of Academic Primary Care, University of Warwick, Coventry, UK. ${ }^{188}$ Adelaide Medical School, University of Adelaide, Adelaide, South Australia, Australia. ${ }^{189}$ Department of Chemistry, University of Porto, Porto, Portugal. ${ }^{190}$ REQUIMTE/LAQV, Porto, Portugal. ${ }^{191}$ Nursing and Health Sciences Department, University of Massachusetts Boston, Boston, MA, USA. ${ }^{192}$ Department of Biostatistics and Epidemiology, University of Oklahoma, Oklahoma City, OK, USA. ${ }^{193}$ Department of Health and Social Affairs, Government of the Federated States of Micronesia, Palikir, Federated States of Micronesia. ${ }^{194}$ Occupational and Environmental Epidemiology Section, Cancer Prevention and Research Institute, Florence, Italy. ${ }^{195}$ Postgraduate Program in Epidemiology, Federal University of Rio Grande do Sul, Porto Alegre, Brazil. ${ }^{196}$ Department of Primary Care and Public Health, Imperial College London, London, UK. ${ }^{197}$ Health Improvement Directorate, Public Health England, London, UK. ${ }^{198}$ School of Public Health, University of Haifa, Haifa, Israel. ${ }^{199}$ School of Public Health and Preventive Medicine, Monash University, Melbourne, Victoria, Australia. ${ }^{200}$ Department of Epidemiology 
and Biostatistics, Zhengzhou University, Zhengzhou, China. ${ }^{201}$ Department of Pharmacology, Tehran University of Medical Sciences, Tehran, Iran. ${ }^{202}$ Obesity Research Center, Research Institute for Endocrine Sciences, Shahid Beheshti University of Medical Sciences, Tehran, Iran. ${ }^{203}$ Department of Radiology, Johns Hopkins University, Baltimore, MD, USA. ${ }^{204}$ Global and Community Mental Health Research Group, University of Macau, Macao, China. ${ }^{205} \mathrm{School}$ of Health and Environmental Studies, Hamdan Bin Mohammed Smart University, Dubai, United Arab Emirates. ${ }^{206}$ Tabriz University of Medical Sciences, Tabriz, Iran. ${ }^{207}$ Biomedical Research Networking Center for Mental Health Network (CIBERSAM), Madrid, Spain. ${ }^{208}$ Research and Development Unit, San Juan de Dios Sanitary Park, Sant Boi De Llobregat, Spain. ${ }^{209}$ School of Nutrition and Food Sciences, Tabriz University of Medical Sciences, Tabriz, Iran. ${ }^{210} \mathrm{Healthcare}$ Management, Maragheh University of Medical Sciences, Maragheh, Iran. ${ }^{211}$ Department of Microbiology, Tehran University of Medical Sciences, Tehran, Iran. ${ }^{212}$ Department of Microbiology, Maragheh University of Medical Sciences, Maragheh, Iran. ${ }^{213}$ School of Nursing and Midwifery Tabriz University of Medical Sciences, Tabriz, Iran. ${ }^{214}$ Independent Consultant, Tabriz, Iran. ${ }^{215}$ Public Health Department, Mizan-Tepi University, Teppi, Ethiopia. ${ }^{216}$ Unit of Epidemiology and Social Medicine, University Hospital Antwerp, Antwerp, Belgium. ${ }^{217}$ School of Public Health, Curtin University, Bentley, Western Australia, Australia. ${ }^{218}$ Medical Biology Research Center, Kermanshah University of Medical Sciences, Kermanshah, Iran. ${ }^{219}$ Population Health, Baker Heart and Diabetes Institute, Melbourne, Victoria, Australia. ${ }^{220}$ Center of Excellence in Behavioral Medicine, Nguyen Tat Thanh University, Ho Chi Minh, Vietnam. ${ }^{221}$ Social Determinants of Health Research Center, Guilan Road Trauma Research Center, Guilan University of Medical Sciences, Rasht, Iran. ${ }^{222}$ Guilan Road Trauma Research Center, Guilan University of Medical Sciences, Rasht, Iran. ${ }^{223}$ Radiology and Nuclear Medicine Department, Kermanshah University of Medical Sciences, Kermanshah, Iran. ${ }^{224}$ Department of Pharmacology and Therapeutics, University of Dhaka, Dhaka, Bangladesh. ${ }^{225}$ Department of Epidemiology and Biostatistics, Tehran University of Medical Sciences, Tehran, Iran.

${ }^{226}$ Computer Science Department, University of Human Development, Sulaimaniyah, Iraq ${ }^{227}$ Department of Internal Medicine, Bucharest Emergency Hospital, Bucharest, Romania. ${ }^{228}$ Faculty of Dentistry, Carol Davila University of Medicine and Pharmacy, Bucharest, Romania. ${ }^{229}$ Clinical Legal Medicine, National Institute of Legal Medicine Mina Minovici, Bucharest, Romania. ${ }^{230}$ Division of Information and Computing Technology, Hamad Bin Khalifa University, Doha, Qatar. ${ }^{231}$ Qatar Foundation for Education, Science and Community Development, Doha, Qatar. ${ }^{232}$ Faculty of Medicine Tunis, Medicine School of Tunis, Baab Saadoun, Tunisia. ${ }^{233}$ Department of Community Medicine, University of Ibadan, Ibadan, Nigeria. ${ }^{234}$ Department of Public Health, Lorestan University of Medical Sciences, Khorramabad, Iran. ${ }^{235}$ Global Health and Development Department, Taipei Medical University, Taipei City, Taiwan. ${ }^{236}$ Research Institute for Endocrine Sciences, Shahid Beheshti University of Medical Sciences, Tehran, Iran. ${ }^{237}$ MRC Epidemiology Unit, University of Cambridge, Cambridge, UK. ${ }^{238}$ Harvard University, Boston, MA, USA. ${ }^{239}$ Institute for Physical Activity and Nutrition, Deakin University, Burwood, Victoria, Australia. ${ }^{240}$ Sydney Medical School, University of Sydney, Sydney, New South Wales, Australia. ${ }^{241}$ Institute of Family Medicine and Public Health, University of Tartu, Tartu, Estonia. ${ }^{242}$ Psychosis Department, Babol Nushirvani University of Technology, Babol, Iran. ${ }^{243}$ Psychiatric Department, Kermanshah University of Medical Sciences, Kermanshah, Iran. ${ }^{244}$ Department of Medical Mycology, Mazandaran University of Medical Sciences, Sari, Iran. ${ }^{245}$ Faculty of Graduate Studies, University of Colombo, Colombo, Sri Lanka. ${ }^{246}$ Institute of Medicine, University of Colombo, Colombo, Sri Lanka. ${ }^{247}$ School of Midwifery, A.T. Still University, Mesa, AZ, USA. ${ }^{248}$ Environmental Research Center, Duke Kunshan University, Kunshan, China. ${ }^{249}$ Department of Medicine, University of Miami, Atlantis, FL, USA. ${ }^{250}$ Department of Ophthalmology, Heidelberg University, Heidelberg, Germany. ${ }^{251}$ Beijing Institute of Ophthalmology, Beijing Tongren Hospital, Beijing, China. ${ }^{252}$ Social Determinants of Health Research Center, University of Social Welfare and Rehabilitation Sciences, Tehran, Iran. ${ }^{253}$ Faculty of Medicine and Health Sciences, University of Opole, Opole, Poland. ${ }^{254}$ Department of Family Medicine and Public Health, University of Opole, Opole, Poland. ${ }^{255}$ Minimally Invasive Surgery Research Center, Iran University of Medical Sciences, Tehran, Iran. ${ }^{256}$ Department of Nutrition and Dietetics, Mekelle University, Mekelle, Ethiopia. ${ }^{257}$ Mazandaran University of Medical Sciences, Sari, Iran. ${ }^{258}$ Isfahan University of Medical Sciences, Isfahan, Iran. ${ }^{259}$ Social Determinants of Health Research Center, Qazvin University of Medical Sciences, Qazvin, Iran. ${ }^{260}$ Department of Epidemiology, Hamadan University of Medical Sciences, Hamadan, Iran. ${ }^{261}$ Research and Development, Australian Red Cross Blood Service, Sydney, New South Wales, Australia. ${ }^{262}$ School of Public Health and Community Medicine, University of New South Wales, Sydney, New South Wales, Australia. ${ }^{263} \mathrm{Hematologic}$ Malignancies Research Center, Tehran University of Medical Sciences, Tehran, Iran. ${ }^{264}$ Hematology-Oncology and Stem Cell Transplantation Research Center, Tehran University of Medical Sciences, Tehran, Iran. ${ }^{265}$ Department of Anesthesiology \& Pain Medicine, University of Washington, Seattle, WA, USA ${ }^{266}$ Odel Campus, University of Nairobi, Nairobi, Kenya. ${ }^{267}$ Michigan State University, East Lansing, MI, USA. ${ }^{268}$ Tabriz Health Management Research Center, Tabriz University of Medical Sciences, Tabriz, Iran. ${ }^{269}$ National Institute for Health Research (NIHR), Tehran University of Medical Sciences, Tehran, Iran. ${ }^{270}$ Department of Public Health and Community Medicine, Jordan University of Science and Technology, Ramtha, Jordan. ${ }^{271}$ Social Determinants of Health Research Center, Ahvaz Jundishapur University of Medical Sciences, Ahvaz, Iran. 272Epidemiology and Biostatistics Department, Health Services Academy, Islamabad, Pakistan. ${ }^{273}$ Population Studies, International Institute for Population Sciences, Mumbai, India. ${ }^{274}$ Department of Internal Medicine, John H. Stroger Jr Hospital of Cook County, Chicago, IL, USA. ${ }^{275}$ Department of Internal Medicine, Dow University of Health Sciences, Karachi, Pakistan. ${ }^{276}$ Institute of Health Policy and Management, Seoul National University, Seoul, South Korea. ${ }^{277}$ Department of Health Policy and Management, Seoul National University, Seoul, South Korea. ${ }^{278}$ Faculty of Health and Wellbeing, Sheffield Hallam University, Sheffield, UK. ${ }^{279}$ Department of Arts and Sciences, Ohio University, Zanesville, OH, USA. ${ }^{280}$ Internal Medicine and Gastroenterology Department, National Hepatology and Tropical Research Institute, Cairo, Egypt. ${ }^{281}$ Department of Medical Parasitology, Cairo University, Cairo, Egypt. ${ }^{282}$ Department of Environmental Health Engineering, Hamadan University of Medical Sciences, Hamadan, Iran. ${ }^{283}$ Department of Public Health, Mazandaran University of Medical Sciences, Sari, Iran. ${ }^{284}$ Department of Nutrition and Health Science, Ball State University, Muncie, IN, USA.
${ }^{285}$ School of Public Health, Kermanshah University of Medical Sciences, Kermanshah, Iran. ${ }^{286}$ School of Medicine, Xiamen University Malaysia, Sepang, Malaysia. ${ }^{287}$ Department of Nutrition, Simmons College, Boston, MA, USA. ${ }^{288}$ Department of Health Management and Health Economics, Kristiania University College, Oslo, Norway. ${ }^{289}$ Department of Health Services Policy and Management, University of South Carolina, Columbia, SC, USA. ${ }^{290}$ Nursing and Health Promotion, Oslo Metropolitan University, Oslo, Norway. ${ }^{291}$ Department of Public Health, Debre Berhan University, Debre Berhan, Ethiopia. ${ }^{292}$ Independent Consultant, Jakarta, Indonesia. ${ }^{293}$ Department of Internal and Pulmonary Medicine, Sheri Kashmir Institute of Medical Sciences, Srinagar, India. ${ }^{294}$ CIBERSAM, San Juan de Dios Sanitary Park, Sant Boi De Llobregat, Spain. ${ }^{295}$ Department of Zoology, University of Oxford, Oxford, UK. ${ }^{296}$ Medical School, Harvard University, Boston, MA, USA. ${ }^{297}$ Department of Anthropology, Panjab University, Chandigarh, India. ${ }^{298}$ Family and Community Health, University of Health and Allied Sciences, Ho, Ghana. ${ }^{299}$ Psychology and Health Promotion, University of Kwazulu-Natal, Durban, South Africa. ${ }^{300}$ Department of Psychiatry, University of Nairobi, Nairobi, Kenya. ${ }^{301}$ Department of Psychology, University College London, London, UK. ${ }^{302}$ International Institute for Population Sciences, Mumbai, India. ${ }^{303}$ Department of Public Health Medicine, University of KwazuluNatal, Durban, South Africa. ${ }^{304}$ Nursing, St John of God Hospital, Duayaw Nkwanta, Ghana. ${ }^{305}$ Nuffield Department of Population Health, University of Oxford, Oxford, UK. ${ }^{306}$ Oxford Biomedical Research Centre, National Institute for Health Research (NIHR), Oxford, UK. ${ }^{307}$ Department of Pediatrics, Post Graduate Institute of Medical Education and Research, Chandigarh, India. ${ }^{308}$ Department of Community and Family Medicine, Academy of Medical Science, Baghdad, Iraq. ${ }^{309}$ Department of Medical Sciences, Uppsala University, Uppsala, Sweden. ${ }^{310}$ Department of Clinical Chemistry and Pharmacology, Uppsala University Hospital, Uppsala, Sweden. ${ }^{311}$ School of Nursing, Hong Kong Polytechnic University, Hong Kong, China. ${ }^{312}$ Department of Medicine, University of Malaya, Kuala Lumpur, Malaysia. ${ }^{313}$ Department of Medicine and Therapeutics, The Chinese University of Hong Kong, Shatin, China. ${ }^{314}$ Department of Dentistry, Radboud University, Nijmegen, The Netherlands. ${ }^{315}$ Section for Translational Health Economics, Heidelberg University Hospital, Heidelberg, Germany. ${ }^{316}$ Department of Vector Biology, Liverpool School of Tropical Medicine, Liverpool, UK ${ }^{317}$ Alliance for Improving Health Outcomes, Quezon City, The Philippines. ${ }^{318}$ Institute of Nutrition, Friedrich Schiller University Jena, Jena, Germany. ${ }^{319}$ Competence Cluster for Nutrition and Cardiovascular Health (NUTRICARD), Jena, Germany. ${ }^{320}$ Physiology Department, Suez Canal University, Ismailia, Egypt. ${ }^{321}$ Proteomics and Metabolomics Unit, Suez Canal University, Ismailia, Egypt. ${ }^{322}$ Department of Cardiology, Damietta University, Damietta, Egypt. ${ }^{323}$ Ophthalmology Department, Aswan Faculty of Medicine, Aswan, Egypt. ${ }^{324}$ Digestive Diseases Research Institute, Tehran University of Medical Sciences, Tehran, Iran. ${ }^{325}$ Non-communicable Diseases Research Center, Shiraz University of Medical Sciences, Shiraz, Iran. ${ }^{326}$ Department of Maternal and Child Nursing and Public Health, Federal University of Minas Gerais, Belo Horizonte, Brazil. ${ }^{327}$ Institute for Social Science Research, The University of Queensland, Brisbane, Queensland, Australia. ${ }^{328}$ Ophthalmology Department, Iran University of Medica Sciences, Tehran, Iran. ${ }^{329}$ Department Ophthalmology, University of Manitoba, Winnipeg, Manitoba, Canada. ${ }^{330}$ Surgery Department, Emergency University Hospital Bucharest, Bucharest, Romania. ${ }^{331}$ Department of Health Education and Health Promotion, Iran University of Medical Sciences, Tehran, Iran. ${ }^{332}$ Campus Caucaia, Federal Institute of Education, Science and Technology of Ceará, Caucaia, Brazil. ${ }^{333}$ Faculty of Health and Education, Botho UniversityBotswana, Gaborone, Botswana. ${ }^{334}$ Division of Plastic Surgery, University of Washington, Seattle, WA, USA. ${ }^{335}$ School of Medicine, University of New South Wales, Sydney, New South Wales, Australia. ${ }^{336}$ Research Department, The George Institute for Global Health, New Delhi, India. ${ }^{337}$ Department of Biology and Biological Engineering, Chalmers University of Technology, Gothenburg, Sweden. ${ }^{338}$ Department of Health Services Research and Policy, London School of Hygiene \& Tropical Medicine, London, UK. ${ }^{339}$ Preventive Oncology Department, National Institute of Cancer Prevention and Research, Noida, India. ${ }^{340}$ Department of Epidemiology and Biostatistics, University of California San Francisco, San Francisco, CA, USA. ${ }^{341}$ Peru Country Office, United Nations Population Fund (UNFPA), Lima, Peru. ${ }^{342}$ Forensic Medicine Division, Imam Abdulrahman Bin Faisal University, Dammam, Saudi Arabia. ${ }^{343}$ Neurocenter, Helsinki University Hospital, Helsinki, Finland. ${ }^{344}$ School of Health Sciences, University of Melbourne, Parkville, Victoria, Australia. ${ }^{345}$ Breast Surgery Unit, Helsinki University Hospital, Helsinki, Finland. ${ }^{346}$ University of Helsinki, Helsinki, Finland. ${ }^{347}$ Clinical Microbiology and Parasitology Unit, Dr Zora Profozic Polyclinic, Zagreb, Croatia. ${ }^{348}$ University Centre Varazdin, University North, Varazdin, Croatia. ${ }^{349}$ Pacific Institute for Research \& Evaluation, Calverton, MD, USA ${ }^{350} \mathrm{Health}$, Evidence and Impact, McMaster University, Hamilton, Ontario, Canada. ${ }^{351}$ Department of Computer Science and Software Engineering, University of Western Australia Perth, Western Australia, Australia. ${ }^{352}$ Department of Public Health, Amrita Institute of Medical Sciences, Kochi, India. ${ }^{353}$ Department of Clinical Biochemistry, Babol University of Medical Sciences, Babol, Iran. ${ }^{354}$ Golestan University of Medical Sciences, Gorgan, Iran. ${ }^{355}$ Foodborne and Waterborne Diseases Research Center, Shahid Beheshti University of Medical Sciences, Tehran, Iran. ${ }^{356}$ Faculty of General Medicine, Kyrgyz State Medical Academy, Bishkek, Kyrgyzstan. ${ }^{357}$ Department of Atherosclerosis and Coronary Heart Disease, National Center of Cardiology and Internal Disease, Bishkek, Kyrgyzstan. ${ }^{358} \mathrm{Health}$ Equity Research Center, Tehran University of Medical Sciences, Tehran, Iran. ${ }^{359}$ Department of Medicine Huddinge, Karolinska Institutet, Stockholm, Sweden. ${ }^{360}$ Department of Food Technology, College of Agriculture, Salahaddin University-Erbil, Erbil, Iraq. ${ }^{361}$ Information Technology Department, University of Human Development, Sulaimaniyah, Iraq. ${ }^{362}$ Department of Biostatistics, Hamadan University of Medical Sciences, Hamadan, Iran. ${ }^{363}$ School of Pharmacy, Haramaya University, Harar, Ethiopia. ${ }^{364}$ Institute of Public Health, Heidelberg University, Heidelberg, Germany. ${ }^{365} \mathrm{Health}$ Systems and Policy Research Unit, Ahmadu Bello University, Zaria, Nigeria. ${ }^{366}$ Faculty of Life Sciences and Medicine, King's College London, London, UK. ${ }^{367}$ Clinical Epidemiology and Public Health Research Unit, Burlo Garofolo Institute for Maternal and Child Health, Trieste, Italy. ${ }^{368} \mathrm{Health}$ Sciences Research Center, Mazandaran University of Medical Sciences, Sari, Iran. ${ }^{369}$ Department of Epidemiology and Biostatistics, Kurdistan University of Medical Sciences, Sanandaj, Iran. ${ }^{370}$ Social Determinants of Health Research Center, Kurdistan University of Medical Sciences, Sanandaj, Iran. ${ }^{371}$ Department of Epidemiology, Iran University of Medical Sciences, Tehran, Iran. ${ }^{372}$ Department of Mathematical Sciences, University of Bath, 
Bath, UK. ${ }^{373}$ International Laboratory for Air Quality and Health, Queensland University of Technology, Brisbane, Queensland, Australia. ${ }^{374}$ Department of Clinical Biochemistry, Tarbiat Modares University, Tehran, Iran. ${ }^{375}$ Department of Health Management and Economics, Tehran University of Medical Sciences, Tehran, Iran. ${ }^{376}$ Federal Institute for Population Research, Wiesbaden, Germany. ${ }^{377}$ Center for Population and Health, Wiesbaden, Germany.

${ }^{378}$ Department of Epidemiology and Biostatistics, University of Gondar, Gondar, Ethiopia. ${ }^{379}$ Department of Pediatric Medicine, Nishtar Medical University, Multan, Pakistan.

${ }^{380}$ Department of Pediatrics \& Pediatric Pulmonology, Institute of Mother \& Child Care, Multan, Pakistan. ${ }^{381}$ Department of Urology, Tehran University of Medical Sciences, Tehran, Iran. ${ }^{382}$ Operating Room Department, Kermanshah University of Medical Sciences, Kermanshah, Iran. ${ }^{383}$ Research and Analytics, Initiative for Financing Health and Human Development, Chennai, India. ${ }^{384}$ Research and Analytics, Bioinsilico Technologies, Chennai, India. ${ }^{385} \mathrm{Cancer}$ Research Center of Cancer Institute, Tehran University of Medical Sciences, Tehran, Iran. ${ }^{386}$ Department of Epidemiology \& Biostatistics, Kermanshah University of Medical Sciences, Kermanshah, Iran. ${ }^{387}$ Suraj Eye Institute, Nagpur, India. ${ }^{388}$ Cochrane South Africa, South African Medical Research Council, Cape Town, South Africa. ${ }^{389}$ Emergency Hospital of Bucharest, Carol Davila University of Medicine and Pharmacy, Bucharest, Romania. ${ }^{390}$ General Surgery Department, Carol Davila University of Medicine and Pharmacy, Bucharest, Romania. ${ }^{391}$ Anatomy and Embryology Department, Carol Davila University of Medicine and Pharmacy, Bucharest, Romania. ${ }^{392}$ Department of Cardiology, Cardio-aid, Bucharest, Romania. ${ }^{393}$ Department of Biological Sciences, University of Embu, Embu, Kenya. ${ }^{394}$ Institute for Global Health Innovations, Duy Tan University, Hanoi, Vietnam. ${ }^{395}$ Center for Excellence in Behavioral Health, Nguyen Tat Thanh University, Ho Chi Minh, Vietnam. ${ }^{396} \mathrm{Global}$ Health Department, University of Washington, Seattle, WA, USA. ${ }^{397}$ Department of Pediatrics, University of Washington, Seattle, WA, USA. ${ }^{398}$ Clinical Pharmacy Unit, Mekelle University, Mekelle, Ethiopia. ${ }^{399}$ Public Health Science Department, School of Public Health and Family Medicine, University of Cape Town, Cape Town, South Africa. ${ }^{400}$ Department of Community and Family Medicine, Iran University of Medical Sciences, Tehran, Iran. ${ }^{401}$ University of Social Welfare and Rehabilitation Sciences, Tehran, Iran. ${ }^{402}$ Department of Health Economics, Tabriz University of Medical Sciences, Tabriz, Iran. ${ }^{403}$ Department of Medicine, University of Cape Town, Cape Town, South Africa. ${ }^{404}$ Centre of Cardiovascular Research and Education in Therapeutics, Monash University, Melbourne, Victoria, Australia. ${ }^{405}$ Independent Consultant, Accra, Ghana. ${ }^{406}$ Translational Health Research Institute, Western Sydney University, Penrith, New South Wales, Australia. ${ }^{407}$ Center for the Aid Program of Research in South Africa (CAPRISA) TB and HIV Pathogenesis Unit, United Nations Programme on HIV/AIDS (UNAIDS), Durban, South Africa. ${ }^{408}$ Department of Psychiatry and Behavioural Neurosciences, McMaster University, Hamilton, Ontario, Canada. ${ }^{409}$ Department of Psychiatry, University of Lagos, Lagos, Nigeria. ${ }^{410}$ Gastroenterology and Liver Disease Research Center, A.C.S. Medical College and Hospital, Tehran, Iran. ${ }^{411}$ Department of Food Science and Postharvest Technology, Gulu University, Gulu, Uganda. ${ }^{412}$ Ghent University, Ghent, Belgium. ${ }^{413}$ Centre for Healthy Start Initiative, Lagos, Nigeria. ${ }^{414}$ Department of Health Promotion and Education, University of Ibadan, Ibadan, Nigeria. ${ }^{415}$ Department of Pharmacology and Therapeutics, University of Nigeria Nsukka, Enugu, Nigeria. ${ }^{416}$ University of Washington, Seattle, WA, USA. ${ }^{417}$ Graduate School of Public Health, San Diego State University, San Diego, CA, USA. ${ }^{418}$ Center for Health Systems Research, National Institute of Public Health, Cuernavaca, Mexico. ${ }^{419}$ School of Medicine, Autonomous University of Madrid, Madrid, Spain. ${ }^{420}$ Department of Nephrology and Hypertension, The Institute for Health Research Foundation Jiménez Díaz University Hospital, Madrid, Spain. ${ }^{421}$ Environmental Management and Toxicology, University of Benin, Benin City, Nigeria. ${ }^{422}$ Faculty of Geoinformation Science and Earth Observation, University of Twente, Enschede, The Netherlands. ${ }^{423}$ Department of Mathematics and Statistics, University of Energy and Natural Resources, Sunyani, Ghana. ${ }^{424}$ Analytical Center, Moscow Institute of Physics and Technology, Dolgoprudny, Russia. ${ }^{425}$ Committee for the Comprehensive Assessment of Medical Devices and Information Technology, Health Technology Assessment Association, Moscow, Russia. ${ }^{426}$ Institute for Advanced Medical Research and Training, University of Ibadan, Ibadan, Nigeria. ${ }^{427}$ Department of Tb \& Respiratory Medicine, Jagadguru Sri Shivarathreeswara University, Mysore, India. ${ }^{428}$ Department of Medicine, Ottawa Hospital Research Institute, Ottawa, Ontario, Canada. ${ }^{429}$ Heidelberg University, Heidelberg, Germany. ${ }^{430}$ Department of Medical Humanities and Social Medicine, Kosin University, Busan, South Korea. ${ }^{431}$ Research and Evaluation, Population Council, New Delhi, India. ${ }^{432}$ Indian Institute of Health Management Research University, Jaipur, India. ${ }^{433}$ Center for Research and Innovation, Ateneo De Manila University, Pasig City, The Philippines. ${ }^{434}$ Department of Genetics, Harvard University, Boston, MA, USA. ${ }^{435}$ Laboratory of Genetics and Molecular Cardiology, University of São Paulo, Sao Paulo, Brazil. ${ }^{436}$ Department of Cardiology, University of Bern, Bern, Switzerland.

${ }^{437}$ Parasitology and Entomology Department, Tarbiat Modares University, Tehran, Iran. ${ }^{438}$ National Centre for Register-Based Research, Aarhus University, Aarhus, Denmark. ${ }^{439}$ Department of Nutrition and Food Sciences, Maragheh University of Medical Sciences, Maragheh, Iran. ${ }^{440}$ Department of Public Health, Maragheh University of Medical Sciences, Maragheh, Iran. ${ }^{441}$ Non-communicable Diseases Research Center, Alborz University of Medical Sciences, Karaj, Iran. ${ }^{442}$ Biomedical Engineering, Amirkabir University of Technology, Tehran, Iran. ${ }^{443}$ College of Graduate Health Sciences, A.T. Still University, Mesa, AZ, USA. ${ }^{444}$ Medichem, Barcelona, Spain. ${ }^{445}$ Molecular and Cell Biology Research Center, Mazandaran University of Medical Sciences, Sari, Iran. ${ }^{446}$ Department of Immunology, Mazandaran University of Medical Sciences, Sari, Iran. ${ }^{447}$ Endocrinology and Metabolism Molecular-Cellular Sciences Institute, Tehran University of Medical Sciences, Tehran, Iran. ${ }^{448}$ Thalassemia and Hemoglobinopathy Research Center, Ahvaz Jundishapur University of Medical Sciences, Ahvaz, Iran. ${ }^{449}$ Department of Clinical Biochemistry, Kermanshah University of Medical Sciences, Kermanshah, Iran. ${ }^{450}$ Sina Trauma and Surgery Research Center, Tehran University of Medical Sciences, Tehran, Iran. ${ }^{451}$ Department of Health Education \& Promotion, Kermanshah University of Medical Sciences, Kermanshah, Iran. ${ }^{452}$ Department of Nephrology, Nizam's Institute of Medical Sciences, Hyderabad, India. ${ }^{453}$ Prevention of Metabolic Disorders Research Center, Shahid Beheshti University of Medical Sciences, Tehran, Iran. ${ }^{454}$ Critical Care Quality Improvement Research Center, Shahid Beheshti University of Medical Sciences, Tehran, Iran. ${ }^{455}$ Policy Research Institute, Kathmandu, Nepal. ${ }^{456}$ Institute for Poverty Alleviation and International Development,
Yonsei University, Wonju, South Korea. ${ }^{457}$ Institute of Public Health, Federal University of Bahia, Salvador, Brazil. ${ }^{458}$ Gonçalo Moniz Institute, Oswaldo Cruz Foundation, Salvador, Brazil. ${ }^{459}$ School of Behavioral Sciences and Mental Health, Iran University of Medical Sciences, Tehran, Iran. ${ }^{460}$ Social Science and Psychology, Western Sydney University, Penrith, New South Wales, Australia. ${ }^{461}$ School of Social Sciences and Psychology, Western Sydney University, Penrith, New South Wales, Australia. ${ }^{462}$ Research Center for Environmental Determinants of Health (RCEDH), Kermanshah University of Medical Sciences, Kermanshah, Iran. ${ }^{463}$ Department of Epidemiology, Shahid Beheshti University of Medical Sciences, Tehran, Iran. ${ }^{464}$ Department of Epidemiology, Birjand University of Medical Sciences, Birjand, Iran. ${ }^{465}$ EPIUnit, University of Porto, Porto, Portugal. ${ }^{466}$ Department of Clinical Research, Federal University of Uberlândia, Uberlândia, Brazil. ${ }^{467}$ Public Health, Addis Ababa University, Addis Ababa, Ethiopia. ${ }^{468}$ Department of Public Health, Wollega University, Nekemte, Ethiopia. ${ }^{469}$ Martin School, University of Oxford, Oxford, UK. ${ }^{470}$ Golestan Research Center of Gastroenterology and Hepatology, Golestan University of Medical Sciences, Gorgan, Iran. ${ }^{471}$ Epidemiology and Biostatistics, Kurdistan University of Medical Sciences, Sanandaj, Iran. ${ }^{472}$ Infectious Diseases and Tropical Medicine Research Center, Babol University of Medical Sciences, Babol, Iran. ${ }^{473}$ School of Biotechnology, Ikiam Amazon Regional University, Tena, Ecuador. ${ }^{474}$ Department of Ocean Science and Engineering, Southern University of Science and Technology, Shenzhen, China. ${ }^{475}$ Department of Biomedical Sciences, University of Sassari, Sassari, Italy. ${ }^{476}$ Department of Health, Safety and Environment (HSE), Shahid Beheshti University of Medical Sciences, Tehran, Iran. ${ }^{477}$ Faculty of Public Health, Kermanshah University of Medical Sciences, Kermanshah, Iran. ${ }^{478}$ Department of Neuroscience, Iran University of Medical Sciences, Tehran, Iran. ${ }^{479}$ Neurogenic Inflammation Research Center, Mashhad University of Medical Sciences, Mashhad, Iran. ${ }^{480}$ Biotechnology Research Center, Mashhad University of Medical Sciences, Mashhad, Iran. ${ }^{481}$ Department of Anatomical Sciences, Kermanshah University of Medical Sciences, Kermanshah, Iran. ${ }^{482}$ Department of Pathology, Al-Imam Mohammad Ibn Saud Islamic University, Riyadh, Saudi Arabia. ${ }^{483}$ School of Health and Policy Management, Faculty of Health, York University, Toronto, Ontario, Canada. ${ }^{484}$ Taleghani Hospital, Kermanshah University of Medical Sciences, Kermanshah, Iran. ${ }^{485}$ Department of Radiology and Nuclear Medicine, Kermanshah University of Medical Sciences, Kermanshah, Iran. ${ }^{486}$ Taleghani Hospital, Kermanshah, Iran. ${ }^{487}$ Center for Health Policy \& Center for Primary Care and Outcomes Research, Stanford University, Stanford, CA, USA. ${ }^{488}$ Department of Entomology, Ain Shams University, Cairo, Egypt. ${ }^{489}$ Centre School of Public Health and Health Management, University of Belgrade, Belgrade, Serbia. ${ }^{490}$ Post-graduate Program in Infectious Diseases and Tropical Medicine, Federal University of Minas Gerais, Belo Horizonte, Brazil. ${ }^{491}$ Department of Community Medicine, PSG Institute of Medical Sciences and Research, Coimbatore, India. ${ }^{492}$ PSG-FAIMER South Asia Regional Institute, Coimbatore, India. ${ }^{493}$ Department of Health and Society, Faculty of Medicine, University of Applied and Environmental Sciences, Bogotá, Colombia. ${ }^{494}$ Faculty of Infectious and Tropical Diseases, London School of Hygiene \& Tropical Medicine, London, UK. ${ }^{495}$ Surgery Department, Hamad Medical Corporation, Doha, Qatar. ${ }^{496}$ Faculty of Health \& Social Sciences, Bournemouth University, Bournemouth, UK. ${ }^{497}$ School of Public Health, Imperial College London, London, UK. ${ }^{498}$ Department of Psychology, University of Alabama at Birmingham, Birmingham, AL, USA. ${ }^{499}$ Center of Expertise in Microbiology, Tehran University of Medical Sciences, Tehran, Iran. ${ }^{500}$ Invasive Fungi Research Center, Mazandaran University of Medical Sciences, Sari, Iran. ${ }^{501}$ Department of Health Promotion and Education, Alborz University of Medical Sciences, Karaj, Iran. ${ }^{502}$ Independent Consultant, Karachi, Pakistan. ${ }^{503}$ School of Medicine, Dezful University of Medical Sciences, Dezful, Iran. ${ }^{504}$ Medical Laboratory Sciences, Mazandaran University of Medical Sciences, Sari, Iran. ${ }^{505}$ Chronic Diseases (Home Care) Research Center, Hamadan University of Medical Sciences, Hamadan, Iran. ${ }^{506}$ Department of Laboratory Sciences, Karaj Islamic Azad University, Kermanshah, Iran. ${ }^{507}$ Department of Basic Sciences, Karaj Islamic Azad University, Kermanshah, Iran. ${ }^{508} \mathrm{HIV} / \mathrm{STI}$ Surveillance Research Center, Kerman University of Medical Sciences, Kerman, Iran. ${ }^{509}$ Policy and Planning Division, Ministry of Health, Riyadh, Saudi Arabia. ${ }^{510}$ University School of Management and Entrepreneurship, Delhi Technological University, New Delhi, India. ${ }^{511}$ Division of General Internal Medicine and Primary Care, Harvard University, Boston, MA, USA. ${ }^{512}$ Usher Institute of Population Health Sciences and Informatics, University of Edinburgh, Edinburgh, UK. ${ }^{513}$ National Institute of Infectious Diseases, Tokyo, Japan. ${ }^{514}$ Finnish Institute of Occupational Health, Helsinki, Finland. ${ }^{515}$ Institute of Medical Epidemiology, Martin Luther University Halle-Wittenberg, Halle, Germany. ${ }^{516}$ Department of Epidemiology, University of Alabama at Birmingham, Birmingham, AL, USA. ${ }^{517}$ Department of Medicine, University of Alabama at Birmingham, Birmingham, AL, USA. ${ }^{518}$ Department of Epidemiology, School of Preventive Oncology, Patna, India. ${ }^{519}$ Department of Epidemiology, Healis Sekhsaria Institute for Public Health, Mumbai, India. ${ }^{520}$ Department of Physiotherapy and Occupational Therapy, Næstved-Slagelse-Ringsted Hospitals, Slagelse, Denmark. ${ }^{521}$ Medical Division, German Leprosy and TB Relief Association Ethiopia, Addis Ababa, Ethiopia. ${ }^{522}$ Department of Medicine, University of Washington, Seattle, WA, USA. ${ }^{523}$ Department of Medicine, University of Calgary, Calgary, Alberta, Canada. ${ }^{524}$ Social Development and Health Promotion Research Center, Kermanshah University of Medical Sciences, Kermanshah, Iran. ${ }^{525} \mathrm{Hospital}$ Universitario de la Princesa, Autonomous University of Madrid, Madrid, Spain. ${ }^{526}$ Centro de Investigación en Red de Enfermedades Respiratorias (CIBERES), Institute of Health Carlos III, Madrid, Spain. ${ }^{527}$ Division of Community Medicine, International Medical University, Kuala Lumpur, Malaysia. ${ }^{528}$ Department of Nursing, Muhammadiyah University of Surakarta, Kartasura, Indonesia. ${ }^{529}$ Department of Community Medicine, Ahmadu Bello University, Zaria, Nigeria. ${ }^{530}$ Department of Criminology, Law and Society, University of California Irvine, Irvine, CA, USA. ${ }^{531}$ Neurology Department, Sree Chitra Tirunal Institute for Medical Sciences and Technology, Trivandrum, India. ${ }^{532}$ Carlos III Health Institute, Biomedical Research Networking Center for Mental Health Network (CIBERSAM), Madrid, Spain. ${ }^{533}$ Department of Medicine, University of Valencia, Valencia, Spain. ${ }^{534}$ School of Social Work, University of Illinois, Urbana, IL, USA. ${ }^{535}$ Cancer Control Center, Osaka International Cancer Institute, Osaka, Japan. ${ }^{536}$ University Institute 'Egas Moniz', Monte Da Caparica, Portugal. ${ }^{537}$ Research Institute for Medicines, Faculty of Pharmacy of Lisbon, University of Lisbon, Lisbon, Portugal. ${ }^{538}$ Department of Pediatrics, King Saud University, Riyadh, Saudi Arabia. ${ }^{539}$ College of Medicine, Alfaisal University, Riyadh, Saudi Arabia. ${ }^{540}$ Anesthesiology Department, University of 
Virginia, Charlottesville, VA, USA. ${ }^{541}$ Syrian Expatriate Medical Association (SEMA), Charlottesville, VA, USA. ${ }^{542}$ Department of Public Health and Community Medicine, Central University Kerala, Kasaragod, India. ${ }^{543}$ Nanyang Technological University, Singapore, Singapore. ${ }^{544}$ School of Exercise and Nutrition Sciences, Queensland University of Technology, Brisbane, Queensland, Australia. ${ }^{545}$ Department of Pathology and Legal Medicine, University of São Paulo, Sao Paulo, Brazil. ${ }^{546}$ Department of Health Economics, Hanoi Medical University, Hanoi, Vietnam. ${ }^{547}$ Department of Molecular Medicine and Pathology, University of Auckland, Auckland, New Zealand. ${ }^{548}$ Clinical Hematology and Toxicology, Military Medical University, Hanoi, Vietnam. ${ }^{549} \mathrm{Gomal}$ Center of Biochemistry and Biotechnology, Gomal University, Dera Ismail Khan. Pakistan. ${ }^{550}$ TB Culture Laboratory, Mufti Mehmood Memorial Teaching Hospital, Dera Ismail Khan, Pakistan. ${ }^{551}$ Division of Health Sciences, University of Warwick, Coventry, UK. ${ }^{552}$ Department of Education and Health, Trauma Research Center, Tehran, Iran. ${ }^{553} \mathrm{Critical}$ and Intensive Care Department, Trauma Research Center, Tehran, Iran. ${ }^{554}$ Argentine Society of Medicine, Buenos Aires, Argentina. ${ }^{555}$ Velez Sarsfield Hospital, Buenos Aires, Argentina. ${ }^{556}$ University Medical Center Groningen, University of Groningen, Groningen, The Netherlands. ${ }^{557}$ Department of General Practice, University Medical Center Groningen, Groningen, The Netherlands. ${ }^{558} \mathrm{Ukk}$ Institute, Tampere, Finland. ${ }^{559} \mathrm{Psych}$ sosocial Injuries Research Center, Ilam University of Medical Sciences, Ilam, Iran. ${ }^{560}$ Raffles Neuroscience Centre, Raffles Hospital, Singapore, Singapore. ${ }^{561}$ Yong Loo Lin School of Medicine, National University of Singapore, Singapore, Singapore. ${ }^{562}$ Department of Medical and Surgical Sciences, University of Bologna, Bologna, Italy. ${ }^{563}$ Occupational Health Unit, Sant'orsola Malpighi Hospital, Bologna, Italy. ${ }^{564}$ Department of Information Technologies and Management, Moscow Institute of Physics and Technology, Dolgoprudny, Russia.

${ }^{565}$ Department of Information and Internet Technologies, I. M. Sechenov First Moscow State Medical University, Moscow, Russia. ${ }^{566}$ Department of Health Care Administration and Economy, National Research University Higher School of Economics, Moscow, Russia. ${ }^{567}$ Foundation University Medical College, Foundation University, Rawalpindi, Pakistan. ${ }^{568}$ Department of Statistics, University of Washington, Seattle, WA, USA. ${ }^{569}$ Department of Epidemiology and Biostatistics, Wuhan University, Wuhan, China. ${ }^{570}$ Department of Psychiatry, University of São Paulo, Sao Paulo, Brazil. ${ }^{571}$ Institute of Child Health, University College London, London, UK. ${ }^{572}$ Cardiology Department, Royal Children's Hospital, Melbourne, Victoria, Australia. ${ }^{573}$ Murdoch Childrens Research Institute, Melbourne, Victoria, Australia. ${ }^{574}$ School of Nursing, Aksum University, Aksum, Ethiopia. ${ }^{575}$ Competence Center of Mortality-Follow-Up, Federal Institute for Population Research, Wiesbaden, Germany. ${ }^{576}$ Cochrane South Africa, Medical Research Council South Africa, Cape Town, South Africa. ${ }^{577}$ Department of Global Health, Stellenbosch University, Cape Town, South Africa.
${ }^{578}$ Department of Pharmacology and Toxicology, Mekelle University, Mekelle, Ethiopia. ${ }^{579}$ Department of Pharmacology, Addis Ababa University, Addis Ababa, Ethiopia. ${ }^{580}$ Zhejiang Spine Research Center, Wenzhou Medical University, Wenzhou, China. ${ }^{581}$ School of Medicine, Nanjing University, Nanjing, China. ${ }^{582}$ Department of Diabetes and Metabolic Diseases, University of Tokyo, Tokyo, Japan. ${ }^{583}$ Department of Health Management, Policy and Economics, Kerman University of Medical Sciences, Kerman, Iran. ${ }^{584}$ Health Services Management Research Center, Kerman University of Medical Sciences, Kerman, Iran. ${ }^{585}$ Department of Pediatrics, University of Jos, Jos, Nigeria. ${ }^{586}$ Department of Pediatrics, Jos University Teaching Hospital, Jos, Nigeria. ${ }^{587}$ Centre for Suicide Research and Prevention, University of Hong Kong, Hong Kong, China. ${ }^{588}$ Department of Social Work and Social Administration, University of Hong Kong, Hong Kong, China. ${ }^{589}$ Department of Psychopharmacology, National Center of Neurology and Psychiatry, Tokyo, Japan. ${ }^{590} \mathrm{Health}$ Economics \& Finance, Global Health, Jackson State University, Jackson, MS, USA. ${ }^{591}$ Research Center for Public Health, Tsinghua University, Peking, China. ${ }^{592}$ Prevention of Cardiovascular Disease Research Center, Shahid Beheshti University of Medical Sciences, Tehran, Iran. ${ }^{593}$ Medical Parasitology Department, Cairo University, Cairo, Egypt. ${ }^{594}$ Global Health Institute, Wuhan University, Wuhan, China. ${ }^{595}$ Department of Health Management and Economics, A.C.S. Medical College and Hospital, Tehran, Iran. ${ }^{596}$ Department of Electrical Engineering, Sharif University of Technology, Tehran, Iran. ${ }^{597}$ Electrical Engineering, Institute for Research in Fundamental Sciences, Tehran, Iran. ${ }^{598}$ Social Determinants of Health Research Center, Ardabil University of Medical Science, Ardabil, Iran. ${ }^{599}$ Maternal and Child Health Division, International Centre for Diarrhoeal Disease Research Bangladesh, Dhaka, Bangladesh. ${ }^{600}$ Department of Medicine, Monash University, Melbourne, Victoria, Australia. ${ }^{601}$ Student Research Committee, Babol University of Medical Sciences, Babol, Iran. ${ }^{602}$ Department of Community Medicine, Ardabil University of Medical Science, Ardabil, Iran. ${ }^{603}$ Social Development and Health Promotion Research Center, Kermanshah University of Medical Sciences, Kermanshah, Iran. ${ }^{604}$ Maternal and Child Wellbeing Unit, African Population Health Research Centre, Nairobi, Kenya. ${ }^{605}$ Public Health Department, Dilla University, Dilla, Ethiopia. ${ }^{606}$ Department of Preventative Medicine, Wuhan University, Wuhan, China. ${ }^{607}$ School of Public Health, Wuhan University of Science and Technology, Wuhan, China. ${ }^{608}$ Indian Institute of Public Health, Public Health Foundation of India, Gurugram, India. ${ }^{609}$ School of BioSciences, University of Melbourne, Parkville, Victoria, Australia. ${ }^{610}$ State University of Semarang, Public Health Science Department, Kota Semarang, Indonesia. ${ }^{611}$ Graduate Institute of Biomedical Informatics, Taipei Medical University, Taipei City, Taiwan. ${ }^{612}$ These authors contributed equally: Roy Burstein, Nathaniel J. Henry. ${ }^{613}$ These authors jointly supervised this work: Christopher J. L. Murray, Simon I. Hay.*e-mail: sihay@uw.edu 


\section{METHODS}

Overview. We fitted a discrete hazards geostatistical model ${ }^{51,52}$ with correlated space-time-age errors and made predictions to generate joint estimates-with uncertainty - of the probability of death (the number of deaths per live births) and the number of deaths for children aged 0-28 days (neonates), children under 1 year old (infants) and children under 5 years old at the subnational level for 99 LMICs for each year from 2000 to 2017. The analytical process is summarized in the flowchart in Extended Data Fig. 6. We made estimates at a grid-cell resolution of approximately $5 \times 5-\mathrm{km}$ and then produced spatially aggregated estimates at the first (that is, states or provinces) and second (that is, districts or counties) administrative levels, as well as the country level.

Countries were selected for inclusion in this study based on their sociodemographic index (SDI) published in the Global Burden of Disease study (GBD $)^{53}$. The SDI is a measure of development based on income per capita, educational attainment and fertility rates among women under 25 years old. We primarily aimed to include all countries in the middle, low-middle or low SDI quintiles, with several exceptions. Brazil and Mexico were excluded despite middle SDI status owing to the availability of high-quality vital registration data in these countries, which have served as the basis for existing subnational estimates of child mortality. Because this study did not incorporate vital registration data sources (see 'Limitations'), Brazil and Mexico were not estimated directly; instead, state-level estimates from the GBD 2017 study were directly substituted in figures where appropriate ${ }^{4}$. Albania and Moldova were excluded despite middle SDI status owing to geographical discontinuity with other included countries and lack of available survey data. North Korea was excluded despite low-middle SDI status owing to geographical discontinuity and insufficient data. As countries with high-middle SDI status in 2017, China and Malaysia were excluded from this analysis. Libya was included despite high-middle SDI status to create better geographical continuity. Island nations with populations under 1 million were excluded because they typically lacked sufficient survey data or geographical continuity for a geospatial analytic approach to be advantageous over a national approach. Supplementary Figure 3.1 shows a map of the countries included in this study and Supplementary Table 3.1 lists the countries.

Data. We extracted individual records from 555 household sample survey and census sources. Records came in the form of either summary birth histories (SBHs) or complete birth histories (CBHs). All input data were subject to quality checks, which resulted in the exclusion of 82 surveys and censuses owing to quality concerns (see Supplementary Information section 3.2 for more details). Data on life and mortality experiences from $\mathrm{CBH}$ sources can be tabulated directly into discrete period and age bins, thus allowing for period-specific mortality estimations, known as the synthetic cohort method ${ }^{54-56}$. For SBH data, we used indirect estimation ${ }^{57}$ to estimate age-specific mortality probabilities and sample sizes and assign them to specific time periods. Complete details are available in Supplementary Information section 3.3.2.

In all cases, after pre-processing, each data point provided a number of deaths and a sample size for an age bin in a specific year and location. We referenced all data points to GPS coordinates (latitude and longitude) wherever possible. In cases in which GPS data were unavailable, we matched data points to the smallest possible areal unit (also referred to as 'polygons'). All polygon data were spatially resampled into multiple GPS coordinates and weighted based on the population distribution following a previously described procedure $e^{5,22,23,58}$ and described in Supplementary Information section 3.4. Our combined global dataset contained approximately 15.9 million births and 1.1 million child deaths. A complete list of data sources is provided in Supplementary Table 8.1.

In addition to data on child mortality, we used a number of spatial data sources for this analysis. These included a suite of geospatial covariates, population estimates and administrative boundaries ${ }^{68}$. These sources and processing procedures are described in Supplementary Information section 4.

Spatial covariates. We extracted values from each of 10 geospatial covariates at each data point location. Geospatial covariates are spatial data represented at the $5 \times 5-\mathrm{km}$ grid-cell resolution. The covariates were travel time to the nearest city, educational attainment of maternal-aged women, the ratio of population of children under 5 to women of reproductive age (ages 15-49 years old), the mass per cubic meter of air of particles with a diameter less than $2.5 \mu \mathrm{m}$, total population, a binary indicator of urbanicity, intensity of lights at night, the proportion of children aged 12-23 months who had received the third dose of diphtheria-pertussistetanus vaccine, incidence rate of Plasmodium falciparum-associated malaria in children under 5 and prevalence of stunting in children under 5 (see Supplementary Information). All covariate values were centred on their means and scaled by their standard deviations. Covariates typically had global spatial coverage and values that vary by year. More details of the spatial covariates can be found in Supplementary Information section 4 .

Analysis. Geostatistical model. To synthesize information across various sources, and to make consistent estimates across space and time, we fitted discrete haz$\operatorname{ards} s^{51,52}$ geostatistical models ${ }^{59}$ to our data. The models were discrete in the sense that ages were represented in seven mutually exclusive bins $(0,1-5,6-11,12-23$, $24-35,36-47$ and $48-59$ months), each with its own assumed constant mortality probability. The model explicitly accounted for variation across age bin, year and space through inclusion of both fixed and random effects. Indicator variables for each age bin were included to form a discrete baseline mortality hazard function, representing the risk of mortality in discrete bins from birth to 59 months of age with covariates set at their means. Baseline hazard functions were allowed to vary in space and time in response to changing covariate values, as well as in response to linear effect on year. To model this relationship, we estimated the effect of each covariate value on the risk of mortality. These estimated effects were then applied to the gridded surface of covariate values to make predictions across the entire study geography. We also included a Gaussian random effect across countries to account for larger-scale variations due to political or institutional effects, as well as a Gaussian random effect for each data source to account for source-specific biases. Finally, we included a Gaussian process random effect with a covariance matrix structured to account for remaining correlation across age, time and physical space. As such, estimates at a specific age, time or place benefitted from drawing predictive strength from data points nearby in all of these dimensions.

For each modelling region, we fitted one such discrete hazards model with a binomial data likelihood. All data were prepared such that we counted or estimated the number of children entering into $(n)$ and dying within $(Y)$ each period-age bin from each GPS-point location $(s)$ in each survey $(k)$ within each country $(c)$.

The number of deaths for children in age band $(a)$ in year $(t)$ at location $(s)$ was assumed to follow a binomial distribution:

$$
Y_{a, s, t} \sim \operatorname{binomial}\left(n_{a, s, t}, P_{a, s, t}\right)
$$

where $P_{a, s, t}$ is the probability of death in age bin $a$, conditional on survival to that age bin for a particular space-time location. Using a generalized linear regression modelling framework, a logit link function is used to relate $P$ to a linear combination of effects:

$$
\operatorname{logit}\left(P_{a, s, t}\right)=\beta^{0}+\sum_{a=2}^{7} I_{a} \beta_{a}^{1}+\beta^{2} X_{s, t}+\beta^{3} t+\nu_{c[s]}+\nu_{k[s]}+Z_{a, s, t}
$$

The first term, $\beta^{0}$, is an intercept, representing the mean for the first age band when all covariates are equal to zero, whereas $\beta_{a}^{1}$ are fixed effects for each age band, representing the mean overall hazard deviation for each age band from the intercept, when all other covariates are equal to zero. $\beta^{2}$ are the effects of geospatial covariates $\left(X_{s, t}\right)$, which we describe in detail in Supplementary Information section 4. $\beta^{3}$ is an overall linear temporal effect to account for overall temporal trends within the region. All geospatial covariates were centred and scaled by subtracting their mean and dividing by their standard deviations. Each $v$ term represents uncorrelated Gaussian random effects: $\nu_{c[s]} \sim \operatorname{normal}\left(0, \sigma_{c}^{2}\right)$ is a country-level random effect applied to all locations $(s)$ within a country $(c) ; \nu_{k}[s] \sim \operatorname{normal}\left(0, \sigma_{k}^{2}\right)$ is a data source-level random effect for the survey $(k)$ from which the data at location $s$ were observed. Data source-level random effects were used to account for systematic variation or biases across data sources and were included in model fitting but not in prediction from fitted models. The term $Z_{a, s, t} \sim \operatorname{Gaussian} \operatorname{process}(0, K)$ is a correlated random effect across age, space and time, and is modelled as a four-dimensional mean zero Gaussian process with covariance matrix $K$. This term accounts for structured residual correlation across these spatial-age-temporal dimensions that are not accounted for by any of the model's other fixed or random effects. This structure was chosen, because the hazard probability for each age group is expected to vary in space and time, and such spatiotemporal correlations are likely to be similar across ages. $K$ is constructed as a separable process across age, space and time $\left(K=\Sigma_{a} \otimes \Sigma_{t} \otimes \Sigma_{s}\right)$. The continuous spatial component is modelled with a stationary isotropic Matérn covariance function, and the age and temporal effects were each assumed to be discrete auto-regressive order 1 . We provide further details on model fitting and specification in Supplementary Information section 5.1.

We assigned priors to all model parameters and performed maximum a posteriori inference using Template Model Builder ${ }^{60}$ software in $\mathrm{R}$ version 3.4. We fitted the model separately for each of 11 world regions (see Supplementary Fig. 3.1), owing to memory constraints and to allow model parameters to vary across epidemiologically distinct regions.

Post-estimation. Using the joint precision matrix and point estimates, we generated 1,000 draws from all model parameters using a multivariate-normal approximation. These model parameter draws were used to predict corresponding draws of mortality probabilities across all age groups for each grid cell in each year. In other words, for each age bin in each year we estimated 1,000 gridded surfaces of mortality probability estimates, each surface corresponding to one draw from the posterior parameter estimates ${ }^{6}$. All subsequent post-estimation procedures were carried out across draws to propagate model uncertainty. We used these estimated 
spatiotemporal gridded surfaces of age-specific mortality probabilities to produce various final resulting data products.

From the fitted model parameters, we produced posterior mortality probability estimates for each age group for each $5 \times 5-\mathrm{km}$ grid cell for each year from 2000 to 2017. We combined gridded age group estimates to obtain infant (under 1) and child (under 5) mortality estimates at each gridded location. Using a conversion from mortality probability to mortality rates, and using a gridded surface of population, we also estimated the number of deaths that occurred in each age group at each location in each year. For both mortality probabilities and counts, we multiplied out corresponding gridded estimates by a constant to ensure that at the national-and in two countries, the first administrative-level unit-aggregated estimates for each age group and year were calibrated such that they equalled estimates in the GBD study ${ }^{4}$. This calibration allowed us to take advantage of national data sources, such as vital registration, that could not be used in this study. We also aggregated grid-cell-level estimates to first and second administrative-level units using gridded population surface to weight estimates. These steps are described in Supplementary Information section 5.2.

Model validation. We used fivefold cross-validation to assess and compare model performance with respect to estimating local trends of age-specific mortality. Each fold was created by combining complete surveys into subsets of approximately $20 \%$ of data sources from the input data. Holding out entire surveys at a time served as a comparable approximation to the type of missingness in our data, essentially helping us check how well our model estimates of mortality probabilities compared to empirical estimates of mortality probability from an unobserved data source that did not inform the model.

For each posterior draw, we aggregated to administrative units. Using data aggregated to the administrative unit and aggregated estimate pairs, we calculated the difference between out-of-sample empirical data estimates and modelled estimates, and we report the following summary metrics: mean error, which serves as a measure of bias, the square root of mean errors, which serves as a measure of the total variation in the errors, the correlation and 95\% coverage. At the second administrative-level unit for under-5 mortality, our out-of-sample $95 \%$ coverage was $93 \%$, correlation was 0.78 , mean absolute error was 0.015 and mean error was -0.0011 . These results indicate a good overall fit, with minimal bias. This procedure and the full validation results are discussed in Supplementary Information section 5.3 .

Limitations. This work should be assessed in full acknowledgement of several data and methodological limitations. We exclusively used $\mathrm{CBH}$ and $\mathrm{SBH}$ data from household survey and census data sources. Ideally, estimates of child mortality should incorporate all available data, including data from administrative vital registration systems. Vital registration systems are commonly present in many middle-income and all high-income countries. There are known data-quality issues with vital registration sources in many middle-income countries ${ }^{48,62}$ that add complications to their inclusion in our modelling procedure. For example, systems may not capture all deaths, and this level of 'underreporting' probably varies in space, time and age. In addition, underreporting is probably negatively correlated with mortality, and could contribute substantial bias to estimates. Statistical methods must be developed to jointly estimate-and adjust for-underreporting in vital registration data before such data can be used in geospatial models of child mortality. Promising work has begun in this domain in specific countries ${ }^{63}$, but further advancement will be necessary to improve estimates across a time series and across many countries at once.

We assume that $\mathrm{SBH}$ and $\mathrm{CBH}$ data were retrospectively representative in the locations in which they were collected. As such, we assume that survey respondents did not migrate. High-spatial-resolution migration estimates with which to adjust estimates do not yet exist, and many of the data sources that we use do not collect information on migration. We conducted a focused sensitivity analysis (Supplementary Information section 5.4.4) for migration in six countries, and found that although our results were generally robust, there was variation by country. Furthermore, despite providing high-quality retrospective data from representative samples of households, birth history data can suffer from certain non-sampling issues, such as survival/selection biases ${ }^{64}$ and misplacement of births ${ }^{65}$. We did not attempt to make corrections to data, and they were used as-is. Furthermore, retrospective birth history data will—by design-have a changing composition of maternal ages depending on the time since the survey. This was minimized by limiting retrospective trends to up to 17 years.

Although we collated a large geo-referenced database of survey data on child mortality, these data represented about $1 \%$ (1.1 million) of total deaths of children under 5 in study areas over the period. Where data do not exist or are not available in certain locations, mean estimates are informed from smoothing to nearby estimates and covariates. As such, there could be additional small-scale heterogeneity that is not picked up by our model. Wider uncertainty intervals in areas with no data account for these potential unknowns, and our $95 \%$ coverage estimates in out-of-sample predictive tests appear to be well-calibrated at the second administrative unit level. Furthermore, discrete localized mortality shock events could be missing in our analysis due to the lack of data and selection biases in surveys and censuses, and spatiotemporal smoothing. Fatal discontinuities are explicitly accounted for at the national or province level by calibration to GBD estimates. In all, $0.35 \%$ ( 0.4 million) of the 123 million deaths over this period were attributed to fatal discontinuities.

On the modelling side, we integrated point and areal data into a continuous model by constructing pseudo-points from areal data. Modelling approaches that integrate point and areal data as part of a joint model likelihood function are in development ${ }^{66}$ but are currently computationally infeasible at the large geographical scales at which we currently model. Furthermore, we divided our models into 11 regional fits (see Supplementary Fig. 3.1), as a full model that encompasses all 99 countries would be computationally infeasible due to memory constraints. Splitting up modelling in this way had the benefit of enabling parameters to vary across epidemiologically distinct world regions. A preferred model, however, would be fitted to all data simultaneously with parameters that are spatially variable.

The separable model used for age-space-time correlations is a common parsimonious assumption afforded in applying spatiotemporal geostatistical models due to efficient computation and inference; however, it yields the assumption of fully symmetric covariance. The symmetry implicit in the separable model dictates, for example, that (holding age constant for simplicity) the covariance between the observations at (location 1, time 1) and (location 2, time 2) is the same as the covariance between (location 1, time 2) and (location 2, time 1). Given our available data density in space-age-time, we believe that attempting to parameterize a more complex non-separable model would be challenging both computationally and inferentially, and it is not clear whether there would be much to benefit from the extra complications.

There are several limitations to address with respect to the use of covariates in the model. Most of the geospatial covariates that we used in the geostatistical model were themselves estimates produced from various geospatial models. Some of those estimated surfaces used covariates that were also included in our model in their estimation process. As such, we emphasize that our model is meant to be predictive, and that drawing inference from fitted coefficients across these highly correlated covariates is problematic and not recommended. Furthermore, we assumed no measurement error in the covariate values and assumed that the functional form between mortality and all covariates was linear in logit space. In certain locations, we used covariate values for prediction that were outside the observed range of the training data. As we explore in Supplementary Information section 5.4.2, however, these areas represent a relatively small proportion of the population.

Finally, we used a method for indirect estimation of SBHs that was recently developed and validated ${ }^{57}$. As such, indirect estimation was carried out as a pre-processing step before fitting the geostatistical model. We attempted to propagate various forms of uncertainty that could be introduced in this step, which resulted in halving the total effective sample size across all SBH data. In future, we aim to fully integrate such processing into the statistical model; such methods are in development ${ }^{67}$, but are not yet computationally feasible at scale.

Reporting summary. Further information on research design is available in the Nature Research Reporting Summary linked to this paper.

\section{Data availability}

The findings of this study are supported by data that are available from public online repositories, data that are publicly available upon request of the data provider and data that are not publicly available due to restrictions by the data provider. Non-publicly available data were used under a license for the current study, but may be available from the authors upon reasonable request and with permission of the data provider. A detailed table of data sources and availability can be found in Supplementary Table 8.1. The full output of the analyses is publicly available in the Global Health Data Exchange (GHDx; http://ghdx.healthdata.org/ record/ihme-data/lmic-under5-mortality-rate-geospatial-estimates-2000-2017) and can be explored using custom data visualization tools (https:/vizhub. healthdata.org/lbd/under5).

51. Lindgren, F., Rue, H. \& Lindström, J. An explicit link between Gaussian fields and Gaussian Markov random fields: the stochastic partial differential equation approach. R. Stat. Soc. 73, 423-498 (2011).

52. Allison, P. D. Discrete-time methods for the analysis of event histories. Sociol. Methodol. 13, 61 (1982).

53. Institute for Health Metrics and Evaluation. Global Burden of Disease Study 2017 (GBD 2017) Socio-Demographic Index (SDI) 1950-2017. http://ghdx. healthdata.org/record/ihme-data/gbd-2017-socio-demographic-index-sdi$1950 \%$ E2\%80\%932017 (GHDx, 2019).

54. Ahmad, O. B., Lopez, A. D. \& Inoue, M. The decline in child mortality: a reappraisal. Bull. World Health Organ. 78, 1175-1191 (2000).

55. Somoza, J. L. Illustrative Analysis: Infant and Child Mortality in Colombia. World Fertility Survey Scientific Report No. 10 (International Statistical Institute, 1980) 
56. Rutstein, S. O. Infant and child mortality: levels, trends and demographic differentials. World Fertility Survey Scientific Report No. 43 (International Statistical Institute, 1984).

57. Burstein, R., Wang, H., Reiner, R. C. Jr \& Hay, S. I. Development and validation of a new method for indirect estimation of neonatal, infant, and child mortality trends using summary birth histories. PLoS Med. 15, e1002687 (2018).

58. Graetz, N. et al. Mapping local variation in educational attainment across Africa. Nature 555, 48-53 (2018).

59. Diggle, P. \& Ribeiro, P. J. Model-based Geostatistics (Springer, 2007).

60. Kristensen, K., Nielsen, A., Berg, C. W., Skaug, H. \& Bell, B. M. TMB: automatic differentiation and Laplace approximation. J. Stat. Softw. 70, 1-21 (2016).

61. Patil, A. P., Gething, P. W., Piel, F. B. \& Hay, S. I. Bayesian geostatistics in health cartography: the perspective of malaria. Trends Parasitol. 27, 246-253 (2011).

62. Phillips, D. E. et al. A composite metric for assessing data on mortality and causes of death: the vital statistics performance index. Popul. Health Metr. 12, 14 (2014).

63. Schmertmann, C. P. \& Gonzaga, M. R. Bayesian estimation of age-specific mortality and life expectancy for small areas with defective vital records. Demography 55, 1363-1388 (2018).

64. Walker, N., Hill, K. \& Zhao, F. Child mortality estimation: methods used to adjust for bias due to AIDS in estimating trends in under-five mortality. PLoS Med. 9, e1001298 (2012).

65. Silva, R. Child mortality estimation: consistency of under-five mortality rate estimates using full birth histories and summary birth histories. PLoS Med. 9, e1001296 (2012).

66. Wilson, K. \& Wakefield, J. Pointless spatial modeling. Biostatistics https://doi. org/10.1093/biostatistics/kxy041 (2018).

67. Wilson, K. \& Wakefield, J. Child mortality estimation incorporating summary birth history data. Preprint at https://arxiv.org/abs/1810.04140 (2018).
68. WorldPop Dataset (WorldPop, accessed 25 July 2017); http://www.worldpop. org.uk/data/get_data/

Acknowledgements This work was primarily supported by grant OPP 1132415 from the Bill \& Melinda Gates Foundation.

Author contributions S.I.H. and R.B. conceived and planned the study. R.B., S.I.H., M.C., N.H., J.L, A.B., N.G. and S.W. identified and obtained data for this analysis. M.C., N.H., J.L, A.B. and S.W. extracted, processed and geo-positioned the data. R.B. and N.H. carried out the statistical analyses with assistance and input from S.I.H., A.O.-Z. and N.M. R.B., N.H., M.C., S.W., L.W., K.J. and L.E. prepared figures and tables. R.B. and L.B.M. wrote the first draft of the manuscript with assistance from S.I.H., S.P., L.E.S. and N.D.W. and all authors contributed to subsequent revisions. All authors provided intellectual input into aspects of this study.

Competing interests This study was funded by the Bill \& Melinda Gates Foundation. Co-authors employed by the Bill \& Melinda Gates Foundation provided feedback on initial maps and drafts of this manuscript. Otherwise, the funders of the study had no role in study design, data collection, data analysis, data interpretation, writing of the final report, or decision to publish. The corresponding author had full access to all of the data in the study and had final responsibility for the decision to submit for publication.

\section{Additional information}

Supplementary information is available for this paper at https://doi.org/ 10.1038/s41586-019-1545-0.

Correspondence and requests for materials should be addressed to S.I.H. Peer review information Nature thanks Paloma Botella and the other, anonymous, reviewer(s) for their contribution to the peer review of this work Reprints and permissions information is available at http://www.nature.com/ reprints. 

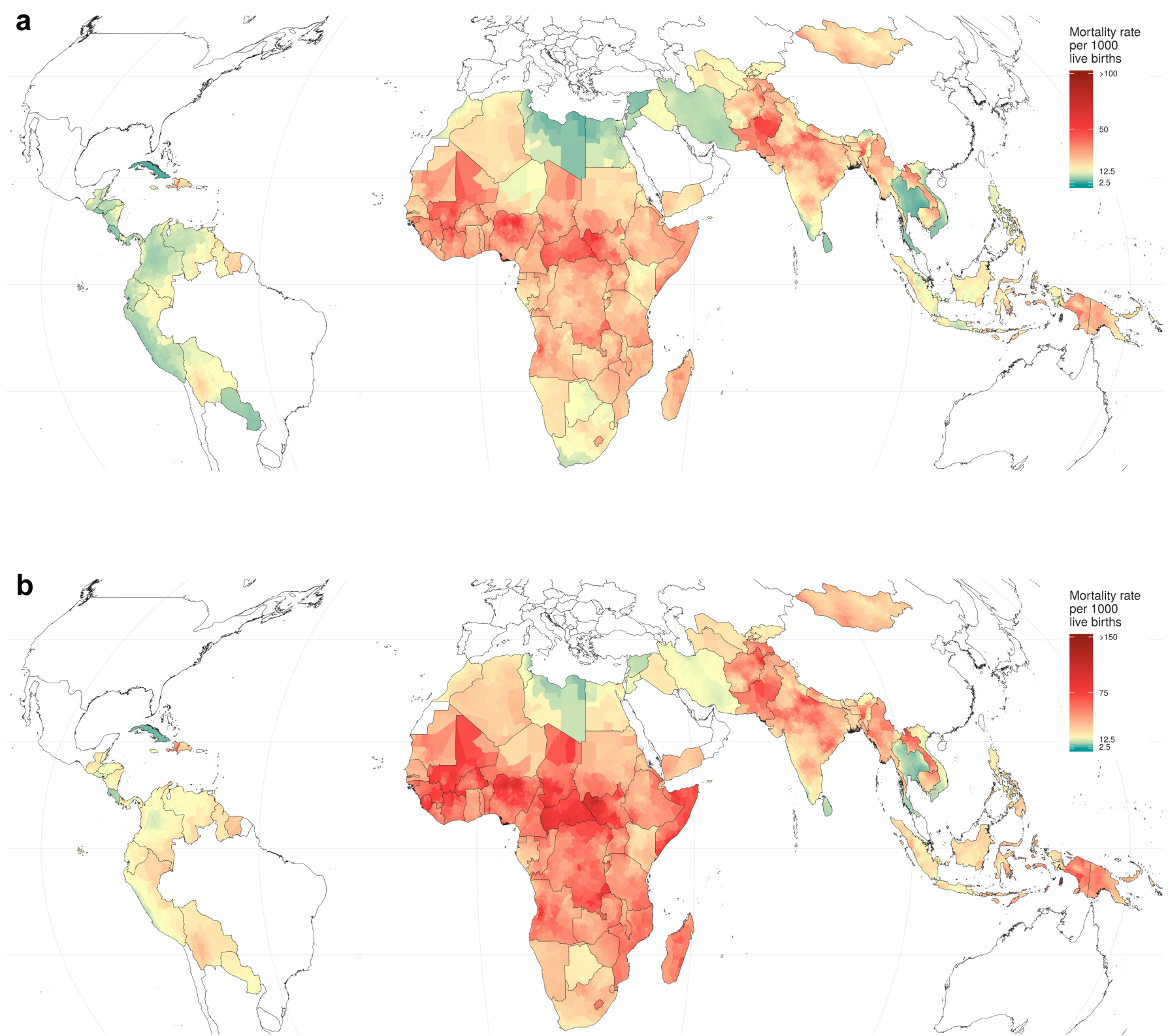

Extended Data Fig. 1 | Neonatal and infant mortality rates in 2017. $\mathbf{a}, \mathbf{b}$, Maps showing the mortality rates of neonates (a; birth to 28 days of units in 2017. Note that the ranges in the keys are different for the two age) and infants (b; under 1 year of age) across second administrative-level maps. 


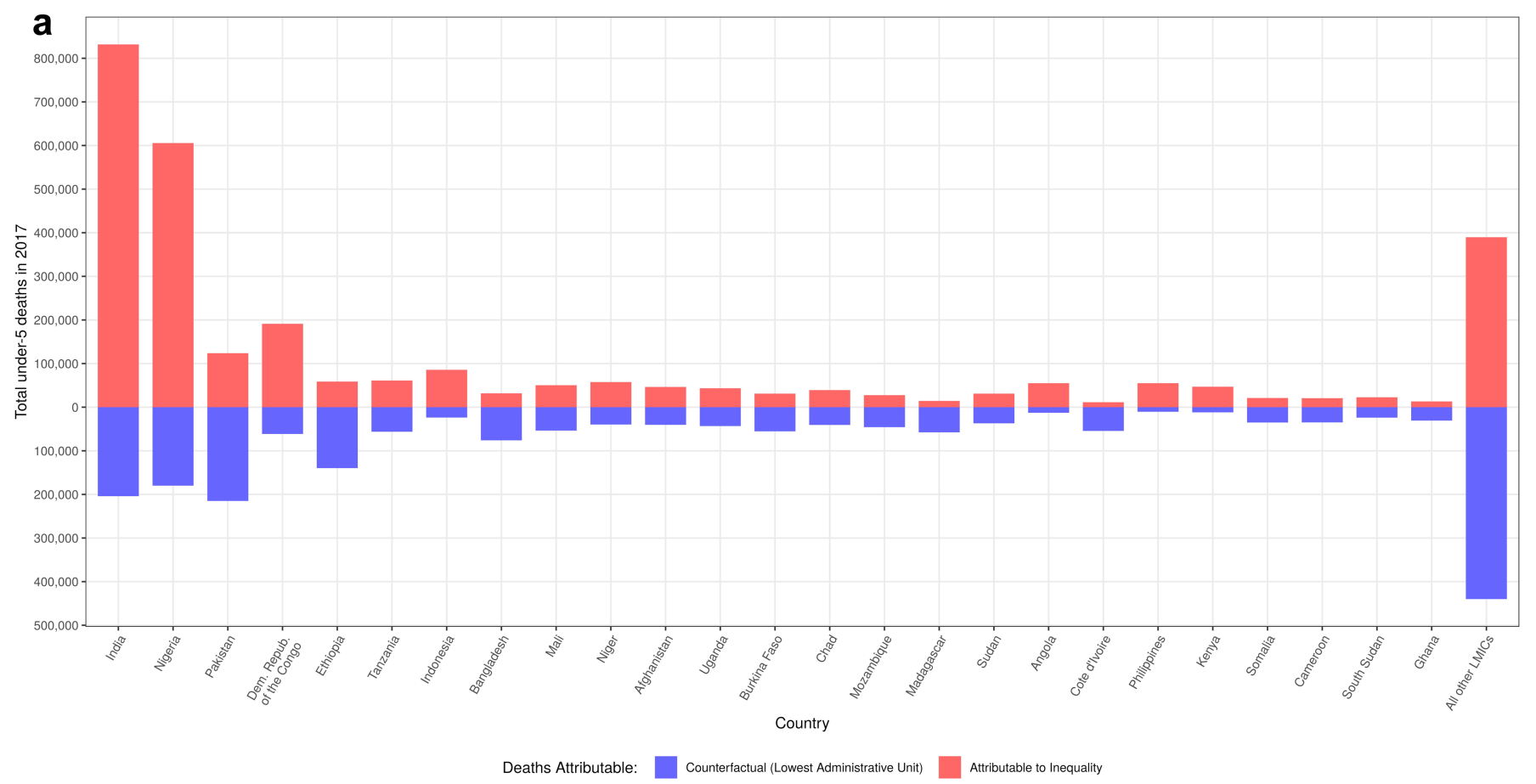

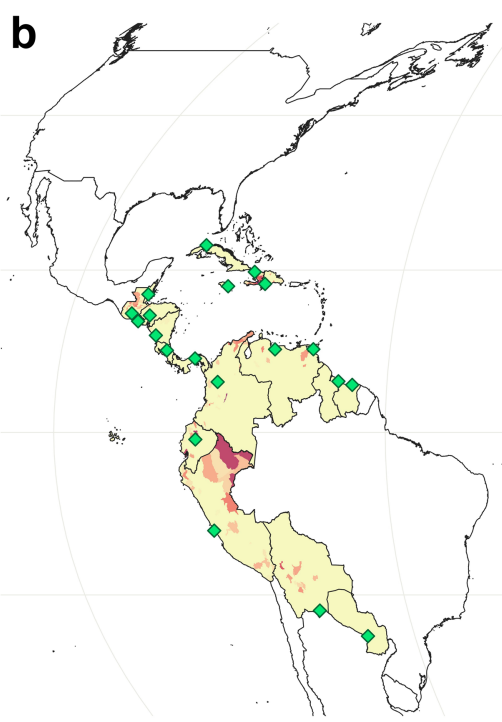

Extended Data Fig. 2 | Impact of inequality on U5MR. a, Potential reduction in the number of deaths that would have occurred if all second administrative-level units in the 20 countries with the greatest number of deaths of children under 5 in 2017 realized a homogenous U5MR that was equal to that of the lowest observed mortality rate in that country. In total, $66 \%$ of under- 5 deaths could have been averted if all countries maintained mortality rates equal to the second administrative-level unit with lowest mortality. If this reference rate is set to the lowest observed rate across all of the 99 countries that were included in this study, $95 \%$ of under- 5 deaths could have been averted. The size of each bar represents the total number

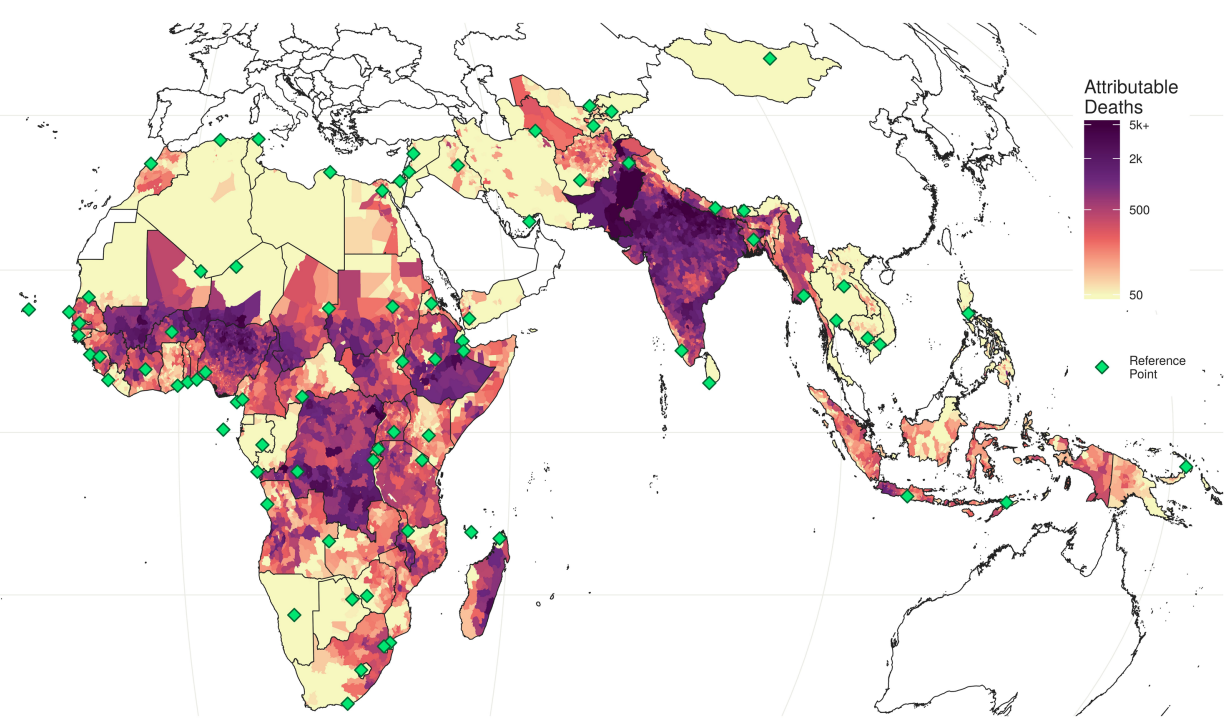

of under- 5 deaths in each country. The red portion of each bar indicates the number of deaths 'attributed' to geographical inequality in mortality rates, whereas the blue portion represents the number of deaths that would remain in the scenario in which all second administrative-level units within countries had the same mortality rate as the best-performing unit. b, Locations of under- 5 deaths 'attributable' to geographical inequality, across all second administrative-level units in each country. Each country has one unit highlighted with a green diamond, which is the reference unit, or the location with the lowest mortality rate in the country in 2017. 


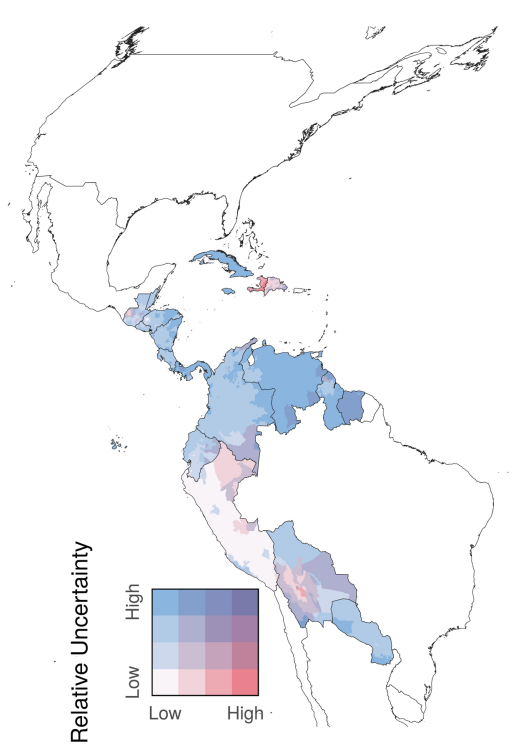

Under-5 Mortality

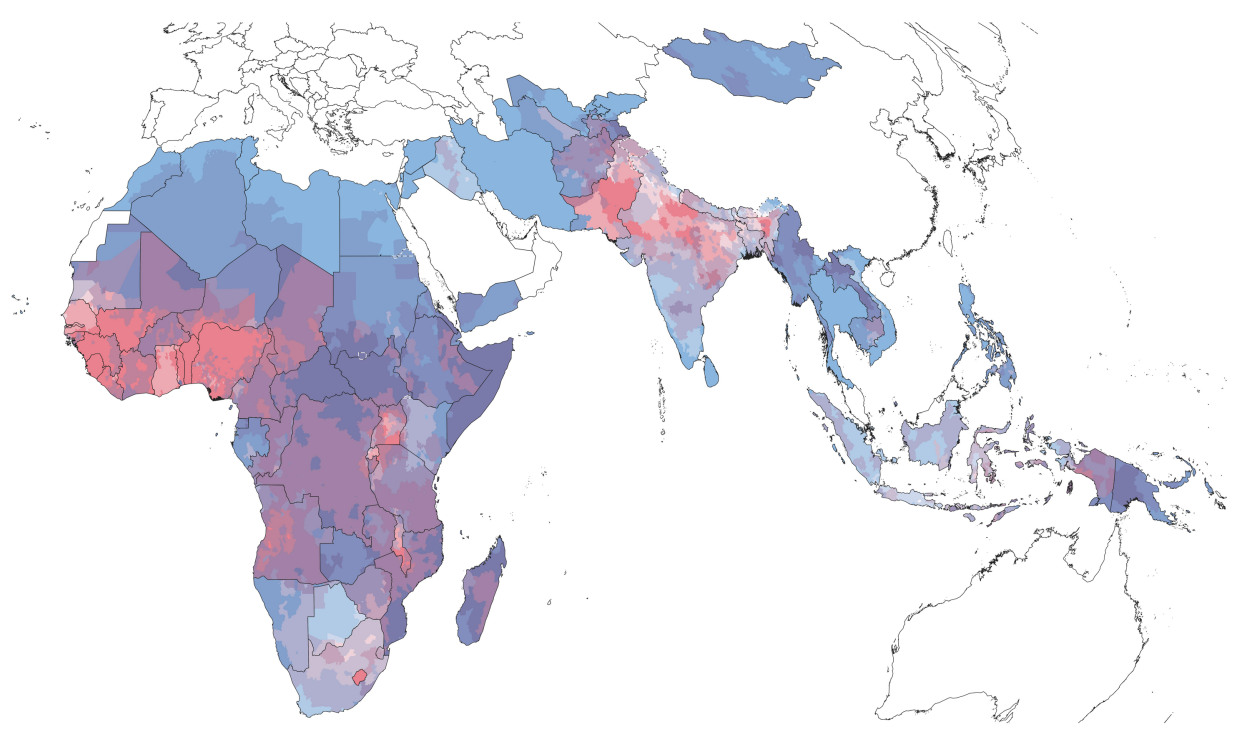

Extended Data Fig. 3 | Relative uncertainty in U5MR estimates for 2017. with mean estimated U5MRs in each second administrative-level unit for 2017. Mean rates and relative uncertainty are split into populationweighted quartiles. These cut-off points indicate the relative uncertainty minimum, 25th, 50th and 75th percentiles, and maximum, which are $0.29,0.51,0.63$ and 0.86 , and 3.12 , respectively. The under- 5 mortality minimum, 25th, 50th and 75th percentiles, and maximum are 1.4, 13.0,

22.9 and 44.8, and 190.6 deaths per 1,000 live births. Areas in which our estimates are more uncertain are coloured with a scale of increasing blue hue, whereas areas in which the mean estimates of U5MR are high are coloured with a scale of increasing red hue. Purple areas have high, but uncertain, estimates of U5MRs. White areas have low relative mortality, with fairly certain estimates. Relative uncertainty is defined as the ratio of the width of the $95 \%$ uncertainty interval to the mean estimate. 
RESEARCH ARTICLE
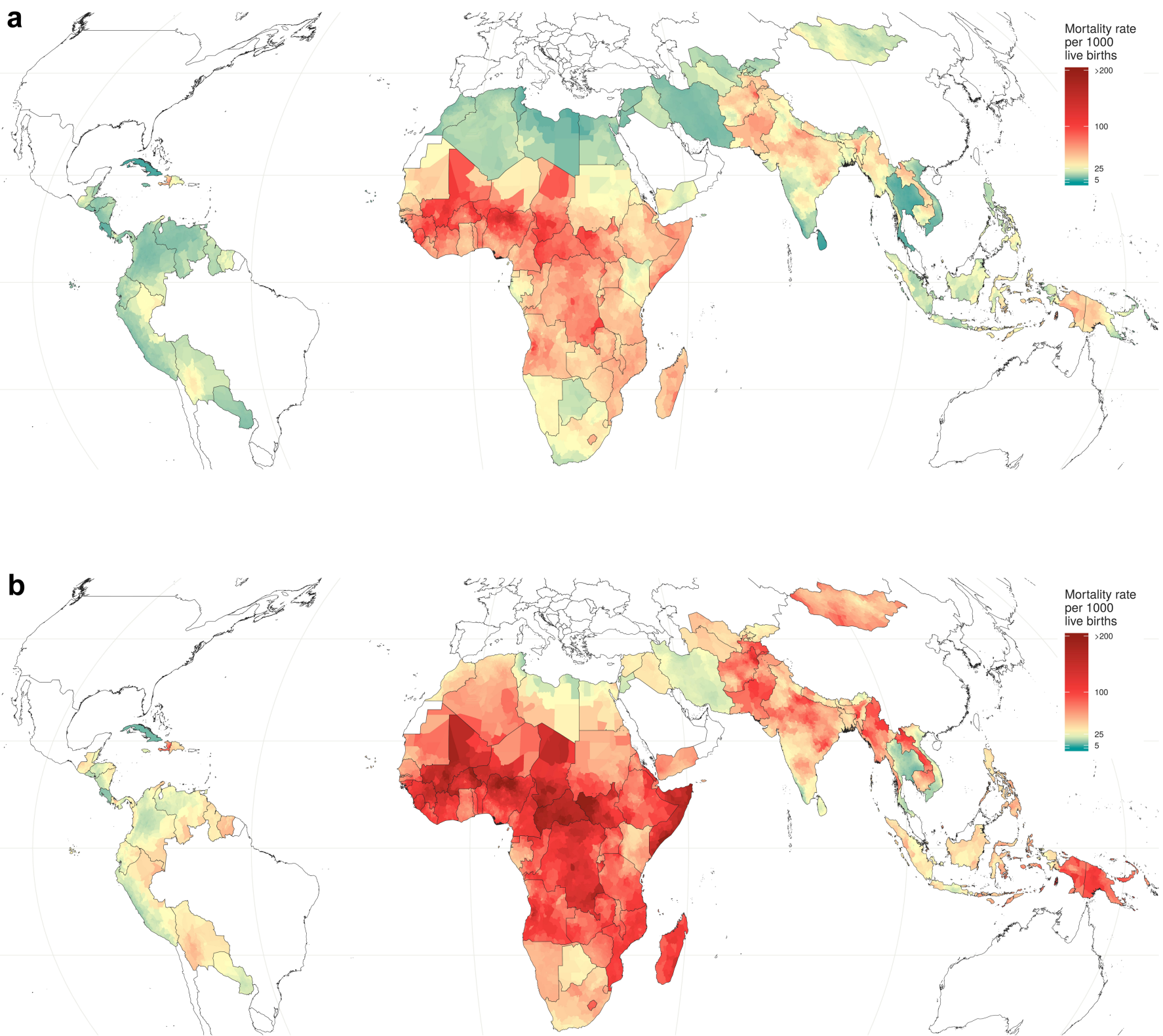

Extended Data Fig. 4 | Lower and upper uncertainty interval boundaries for U5MR mortality estimates in 2017. a, b, Lower (a) and upper (b) 95\% uncertainty intervals for U5MR estimates across the second administrative-level units in 99 countries. 


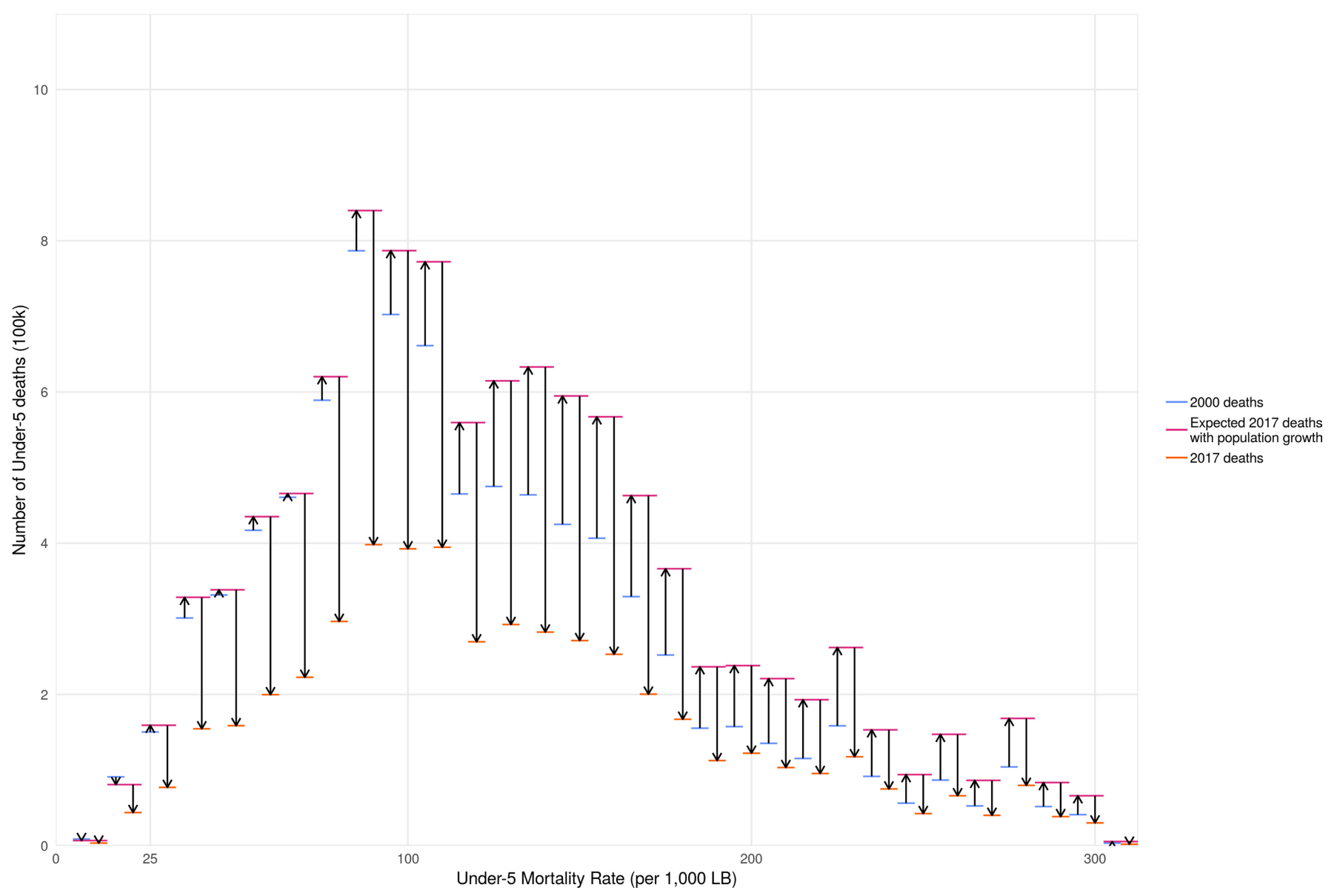

Extended Data Fig. 5 | The counteracting forces of population change and mortality rate decline on total number of under-5 deaths. Arrow plots show the mortality rate strata (bins of 10 per 1,000 livebirths) in 2000. 


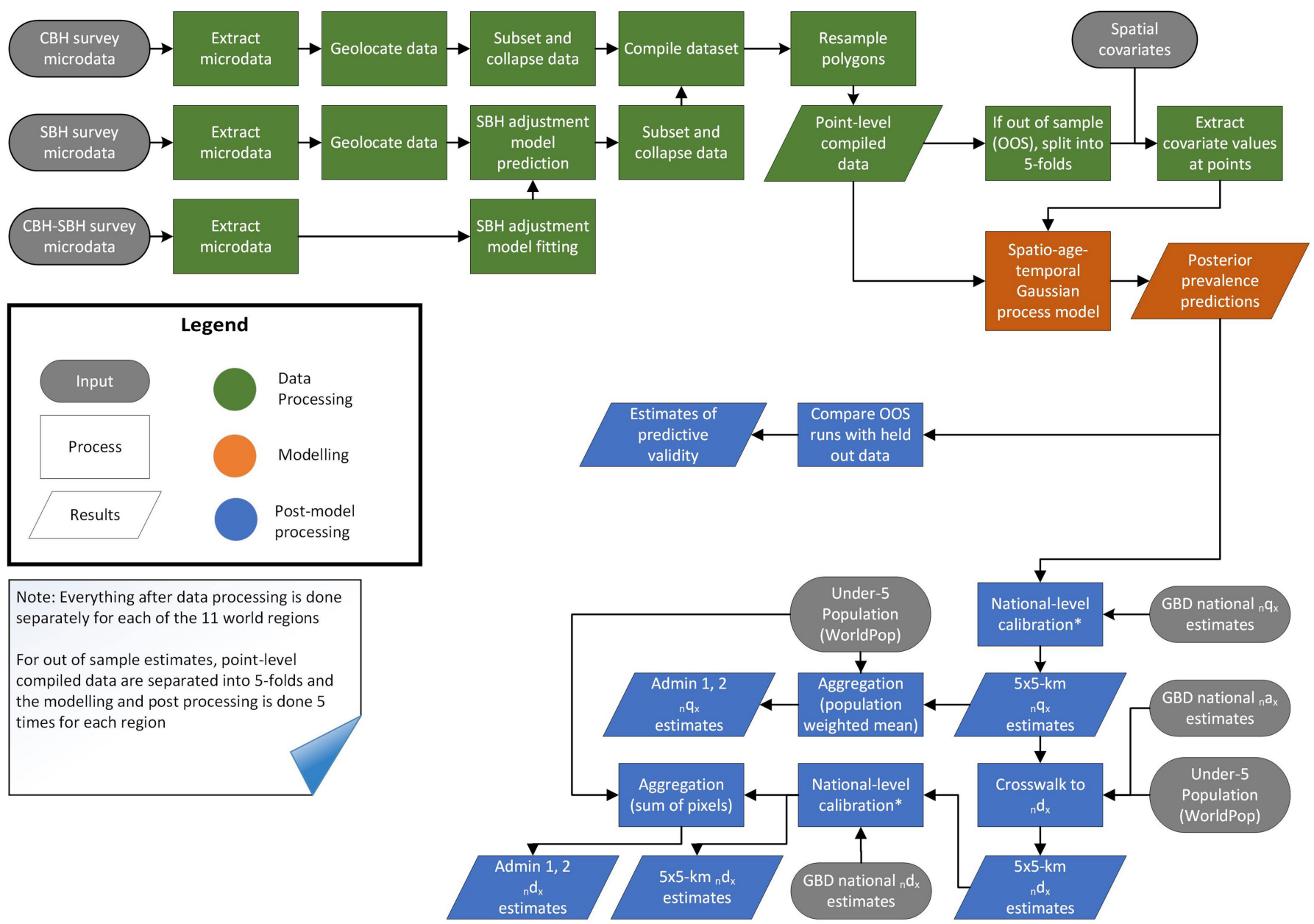

Extended Data Fig. 6 | Flowchart summarizing analytical process. Standard demographic notation were used. $n$, length of age bin; $x$, starting age of age bin; $d$, number of deaths; $q$, probability of death; $a$, average time lived in age bin by those who died in the age bin. 


\section{nature research}

Corresponding author(s): Simon Hay

Last updated by author(s): Jul 9, 2019

\section{Reporting Summary}

Nature Research wishes to improve the reproducibility of the work that we publish. This form provides structure for consistency and transparency in reporting. For further information on Nature Research policies, see Authors \& Referees and the Editorial Policy Checklist.

\section{Statistics}

For all statistical analyses, confirm that the following items are present in the figure legend, table legend, main text, or Methods section.

$\mathrm{n} / \mathrm{a}$ Confirmed

\ The exact sample size $(n)$ for each experimental group/condition, given as a discrete number and unit of measurement

$\square$ A statement on whether measurements were taken from distinct samples or whether the same sample was measured repeatedly

$\triangle \square$ The statistical test(s) used AND whether they are one- or two-sided

Only common tests should be described solely by name; describe more complex techniques in the Methods section.

$\searrow$ A description of all covariates tested

$\square \bigotimes$ A description of any assumptions or corrections, such as tests of normality and adjustment for multiple comparisons

$\checkmark$ A full description of the statistical parameters including central tendency (e.g. means) or other basic estimates (e.g. regression coefficient)

AND variation (e.g. standard deviation) or associated estimates of uncertainty (e.g. confidence intervals)

$\square$ For null hypothesis testing, the test statistic (e.g. $F, t, r$ ) with confidence intervals, effect sizes, degrees of freedom and $P$ value noted

Х $\square$ Give $P$ values as exact values whenever suitable.

$\square$ For Bayesian analysis, information on the choice of priors and Markov chain Monte Carlo settings

$\bigotimes \square$ For hierarchical and complex designs, identification of the appropriate level for tests and full reporting of outcomes

$\bigotimes \square$ Estimates of effect sizes (e.g. Cohen's $d$, Pearson's $r$ ), indicating how they were calculated

Our web collection on statistics for biologists contains articles on many of the points above.

\section{Software and code}

Policy information about availability of computer code

Data collection No primary data collection was carried out for this analysis.

Data analysis This analysis was carried out using R version 3.5.0. The main statistical model used the Template Model Builder (TMB) software version 1.7.14 in R. All code used for these analyses is publicly available online at https://github.com/ihmeuw/lbd/tree/u5mr-2019.

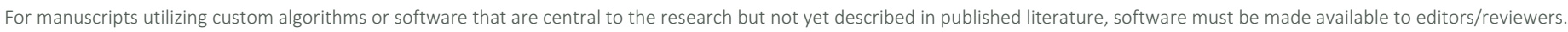
We strongly encourage code deposition in a community repository (e.g. GitHub). See the Nature Research guidelines for submitting code \& software for further information.

\section{Data}

Policy information about availability of data

All manuscripts must include a data availability statement. This statement should provide the following information, where applicable:

- Accession codes, unique identifiers, or web links for publicly available datasets

- A list of figures that have associated raw data

- A description of any restrictions on data availability

The findings of this study are supported by data available in public online repositories, data publicly available upon request of the data provider, and data not publicly available due to restrictions by the data provider. Non-publicly available data were used under license for the current study but may be available from the authors upon reasonable request and with permission of the data provider. A detailed table of data sources and availability can be found in Supplementary Table 8.1. The full output of the analyses are publicly available in the Global Health Data Exchange (http://ghdx.healthdata.org/) and can further be explored via data visualization tools (https://healthdata.org/lbd/under5). 
Please select the one below that is the best fit for your research. If you are not sure, read the appropriate sections before making your selection.

$\bigotimes$ Life sciences

Behavioural \& social sciences

Ecological, evolutionary \& environmental sciences

For a reference copy of the document with all sections, see nature.com/documents/nr-reporting-summary-flat.pdf

\section{Life sciences study design}

All studies must disclose on these points even when the disclosure is negative.

Sample size This observational study incorporated all available survey data sources that met the inclusion criteria as described in the Methods section. The combined dataset used in this analysis contained 25.1 million births and 1.3 million child deaths.

Data exclusions Surveys were excluded due to missingness greater than $10 \%$ in date of birth or death and children ever born or died, unrealistic geographic trends compared to other surveys in nearby country-years, inability to match the microdata to geographic locations, or non-standard methodology. A full list of excluded surveys is included in Supplementary Table 8.2.

Replication This is an observational study using many years of survey and surveillance data and could be replicated.

Randomization This analysis is an observational mapping study, there were no experimental groups.

Blinding Blinding was not relevant to this study, as it was an observational study using survey and surveillance data.

\section{Reporting for specific materials, systems and methods}

We require information from authors about some types of materials, experimental systems and methods used in many studies. Here, indicate whether each material, system or method listed is relevant to your study. If you are not sure if a list item applies to your research, read the appropriate section before selecting a response.

\begin{tabular}{|c|c|c|c|}
\hline \multicolumn{2}{|c|}{ Materials \& experimental systems } & \multicolumn{2}{|c|}{ Methods } \\
\hline $\mathrm{n} / \mathrm{a}$ & Involved in the study & $\mathrm{n} / \mathrm{a}$ & Involved in the study \\
\hline 凶 & Antibodies & Х & $\square$ ChIP-seq \\
\hline$\bigotimes$ & $\square$ Eukaryotic cell lines & Х & $\square$ Flow cytometry \\
\hline$凶$ & $\square$ Palaeontology & Х & $\square$ MRI-based neuroimaging \\
\hline$\bigotimes$ & $\square$ Animals and other organisms & & \\
\hline$\bigotimes$ & $\square$ Human research participants & & \\
\hline Х & $\square$ Clinical data & & \\
\hline
\end{tabular}

